\title{
Multicast Capacity for Large Scale Wireless Ad Hoc Networks
}

\author{
Xiang-Yang $\mathrm{Li}$ * \\ Dept. of Computer Science \\ Illinois Institute of Technology \\ Chicago, IL 60616, USA \\ xli@cs.iit.edu
}

\author{
Shao-Jie Tang \\ Dept. of Computer Science \\ Illinois Institute of Technology \\ Chicago, IL 60616, USA \\ stang7@iit.edu
}

\author{
Ophir Frieder \\ Dept. of Computer Science \\ Illinois Institute of Technology \\ Chicago, IL 60616, USA \\ ophir@cs.iit.edu
}

\begin{abstract}
In this paper, we study the capacity of a large-scale random wireless network for multicast. Assume that $n$ wireless nodes are randomly deployed in a square region with side-length $a$ and all nodes have the uniform transmission range $r$ and uniform interference range $R>r$. We further assume that each wireless node can transmit/receive at $W$ bits/second over a common wireless channel. For each node $v_{i}$, we randomly pick $k-1$ nodes from the other $n-1$ nodes as the receivers of the multicast session rooted at node $v_{i}$. The aggregated multicast capacity is defined as the total data rate of all multicast sessions in the network. In this paper we derive matching asymptotic upper bounds and lower bounds on multicast capacity of random wireless networks. We show that the total multicast capacity is $\Theta\left(\sqrt{\frac{n}{\log n}} \cdot \frac{W}{\sqrt{k}}\right)$ when $k=O\left(\frac{n}{\log n}\right)$; the total multicast capacity is $\Theta(W)$ when $k=\Omega\left(\frac{n}{\log n}\right)$. Our bounds unify the previous capacity bounds on unicast (when $k=2$ ) by Gupta and Kumar [7] and the capacity bounds on broadcast (when $k=n)$ in $[11,20]$. We also study the capacity of group-multicast for wireless networks where for each source node, we randomly select $k-1$ groups of nodes as receivers and the nodes in each group are within a constant hops from the group leader. The same asymptotic upper bounds and lower bounds still hold. For arbitrary networks, we provide a constructive lower bound $\Omega\left(\frac{\sqrt{n}}{\sqrt{k}} \cdot W\right)$ for aggregated multicast capacity when we can carefully place nodes and schedule node transmissions.
\end{abstract}

\section{Categories and Subject Descriptors}

C.2.1 [Network Architecture and Design]: Wireless communication, Network topology; G.2.2 [Graph Theory]: Network problems, Graph algorithms

\section{General Terms}

Algorithms, Design, Theory

${ }^{*}$ The work of the author is partially supported by NSF CCR0311174. Part of the work was done when the author visited Microsoft Research Asia, BeiJing, China.

Permission to make digital or hard copies of all or part of this work for personal or classroom use is granted without fee provided that copies are not made or distributed for profit or commercial advantage and that copies bear this notice and the full citation on the first page. To copy otherwise, to republish, to post on servers or to redistribute to lists, requires prior specific permission and/or a fee.

MobiCom'07, September 9-14, 2007, Montréal, Québec, Canada. Copyright 2007 ACM 978-1-59593-681-3/07/0009 ...\$5.00

\section{Keywords}

Wireless ad hoc networks, capacity, multicast, broadcast, unicast, scheduling, optimization, probability theory.

\section{INTRODUCTION}

In wireless ad hoc networks, wireless nodes may cooperate in routing each others' packets. Lack of a centralized control of the functionality and possible node mobility give rise to many challenging issues at the network layer, the medium access layer, and physical layer of a wireless ad hoc network. At the network layer, the main challenging problem is that of routing, which has to deal with time-varying network topology, possible power-constraints of wireless nodes, and the characteristics of the wireless channel (such as unstable, broadcast nature, fading and so on). The choice of medium access control is also restricted by the fact that the network topology is time-varying, and there is no centralized control. In the literature, a number of results have been proposed to use the TDMA, CDMA, FDMA, and the dynamic assignment of frequency bands to improve the network throughput. Notice that TDMA has recently been proposed to improve the network throughput for some networks or partial of the networks $[1,23]$, especially for static networks. At the physical layer an important issue is the power-control, which has been studied extensively in the literature. A careful selection of the transmission power of nodes can not only improve the nodal life, but also improve the spatial reuse of frequency and consequently possibly improve the network throughput.

In many applications, e.g., wireless sensor networks, we often need a rough estimation on the achievable throughput when we randomly deploy $n$ wireless nodes in a given region. The main purpose of this paper is to study the asymptotic capacity of large scale random wireless networks when we choose the best protocols for all layers. As in the literature, we will mainly consider one type of networks, large scale random networks, where a large number of nodes are randomly placed in the deployment region. We will study the capacity of a given wireless network where the nodes positions are given a priori, and how the capacity of wireless networks scale with the number of nodes in the networks (when given a fixed deployment region), or scale with the size of the deployment region (when given a fixed deployment density) for multicast. We assume that a set of $n$ wireless nodes $V=\left\{v_{1}, v_{2}, \cdots, v_{n}\right\}$ are randomly distributed (with uniform distribution) in a square region with a side-length $a$ and all nodes have the same transmission range $r$. For most results presented in this paper, we assume that values of $a$ and $r$ are selected such that the resulted network will be connected with high probability (w.h.p.). The results derived under this model also imply the same results for the dense model, when $n$ nodes are distributed in a fixed region (such as a unit square by a 
proper scaling) and the uniform transmission range of all nodes are selected as the critical transmission range (CTR) to get a connected network with high probability.

In this paper, we will concentrate on the multicast capacity of a random wireless network, which generalizes both the unicast capacity [7] and broadcast capacity [11,20] for random networks. Assume that a subset $\mathcal{S} \subseteq V$ of $n_{s}=|\mathcal{S}|$ nodes will serve as the source nodes of $n_{s}$ multicast sessions. The most results in this paper assume that $\mathcal{S}=V$. Each node $v_{i} \in \mathcal{S}$ has a set of randomly chosen $n_{d}=k-1$ destination nodes to which it wishes to send data at an arbitrary data rate $\lambda_{i}$. The multicast capacity of a random network is defined as $\Lambda_{k}(n)=\sum_{i=1}^{n} \lambda_{i}$ when there is a schedule of transmissions such that all multicast flows will be received by their destination nodes successfully within a finite delay. To describe when a transmission is received successfully by its intended recipient, we will allow one possible model for a successful one-hop reception: protocol model. We assume that each node $v \in V$ has a fixed constant transmission range $r$ and a fixed constant interference range $R>r$. A node $u$ can successfully receive a transmission from another node $v$ with $\|v-u\| \leq r$ iff there is no other node $w$ such that $\|w-u\| \leq R$ and node $w$ is transmitting simultaneously with node $v$. Here $\|w-u\|$ is the Euclidean distance between $w$ and $u$.

We assume the following simple wireless channel model as in the literature: each wireless node can transmit at $W$ bits/second over a common wireless channel. For presentation simplicity, we assume that there is only one channel in the wireless networks. We will see that it is immaterial to results presented in this paper if the channel is broken up into several sub-channels of capacity $W_{1}, W_{2}, \cdots$, $W_{M}$ bits/second as long as we have $\sum_{i=1}^{M} W_{i}=W$. As always, we assume that the packets are sent from node to node in a multihop manner until they reach their final destinations. The packets could be buffered at intermediate nodes while awaiting for transmission. In this paper, we assume that the buffer is large enough so packets will not get dropped by any intermediate node. We leave it as future work to study the scenario when the buffers of intermediate nodes are bounded by some values. In some results, we assume that every intermediate node have infinite buffer size. For most of the results presented here, the delay of the routing is not considered, i.e., the delay in the worst case could be arbitrarily large for some results.

Our Main Contributions: We propose two regimes for multicast capacity in terms of $k$. We derive matching analytical upper bounds and lower bounds on multicast capacity of a random wireless network. Assume that the side-length $a$ of the deployment square and the transmission range $r$ are selected such that the network is connected almost surely i.e., $\frac{a}{r}=\Theta\left(\sqrt{\frac{n}{\log n}}\right)$. We show that the aggregated multicast capacity of $n$ random multicasts is

$$
\Lambda_{k}(n)= \begin{cases}\Theta\left(\sqrt{\frac{n}{\log n}} \cdot \frac{W}{\sqrt{k}}\right) & \text { when } k=O\left(\frac{n}{\log n}\right), \\ \Theta(W) & \text { when } k=\Omega\left(\frac{n}{\log n}\right)\end{cases}
$$

Our bounds unify the previous capacity bounds on unicast (when $k=2$ ) by Gupta and Kumar [7] and the capacity bounds on broadcast (when $k=n$ ) in $[11,20]$. Consequently, the per-node multicast capacity $\lambda_{k}(n)$ of $n$ multicast sessions (with $k-1$ receivers per multicast session) is

$$
\lambda_{k}(n)= \begin{cases}\Theta\left(\sqrt{\frac{1}{n \log n}} \cdot \frac{W}{\sqrt{k}}\right) & \text { when } k=O\left(\frac{n}{\log n}\right), \\ \Theta\left(\frac{W}{n}\right) & \text { when } k=\Omega\left(\frac{n}{\log n}\right)\end{cases}
$$

The above capacity bounds are implied by a more general result for the following network setting there are $n_{s}$ multicast ses- sions, each with $k-1$ randomly selected receivers from $V$, and the transmission range $r$ and side-length $a$ of the deployment square satisfying that the resulted random network is connected with high probability. Generally, when $\lim _{n \rightarrow \infty} n_{s} \cdot k=\infty$, we prove that the aggregated multicast capacity of $n_{s}$ multicast sessions is

$$
\Lambda_{k}(n)= \begin{cases}\Theta\left(\frac{a}{r} \cdot \frac{W}{\sqrt{k}}\right) & \text { when } k=O\left(\frac{a^{2}}{r^{2}}\right) \\ \Theta(W) & \text { when } k=\Omega\left(\frac{a^{2}}{r^{2}}\right)\end{cases}
$$

and the per-source multicast capacity of $n_{s}$ multicast sessions is

$$
\lambda_{k}(n)= \begin{cases}\min \left(W, \Theta\left(\frac{a}{r} \cdot \frac{W}{n_{s} \sqrt{k}}\right)\right) & \text { when } k=O\left(\frac{a^{2}}{r^{2}}\right) \\ \Theta\left(\frac{W}{n_{s}}\right) & \text { when } k=\Omega\left(\frac{a^{2}}{r^{2}}\right)\end{cases}
$$

We also study the multicast capacity for group-multicast where, for each source node, we randomly select $k-1$ groups of nodes as receivers and the nodes in each group are within a constant number of hops from the group leader. We show that the asymptotic multicast capacity is still $\Theta\left(\sqrt{\frac{n}{\log n}} \cdot \frac{W}{\sqrt{k}}\right)$ when $k=O\left(\frac{n}{\log n}\right)$; and is $\Theta(W)$ when $k=\Omega\left(\frac{n}{\log n}\right)$. For multicast in arbitrary networks, we provide a constructive lower bound $\Omega\left(\frac{\sqrt{n}}{\sqrt{k}} \cdot W\right)$ when we can carefully place nodes and schedule node transmissions.

The rest of the paper is organized as follows. In Section 2 we discuss in detail the network model and the channel model used in this paper. In Section 3, we first present some upper-bounds on multicast capacity for random networks. In Section 4, we then present an efficient method for multicast and prove that the capacity achieved by this method asymptotically matches the upper-bounds derived before. In Section 5, we study the multicast capacity bounds for group-multicast and the multicast capacity bounds for arbitrarily networks. We review the related results on network capacities in Section 6 and conclude the paper in Section 7 with the discussion of some possible future works.

\section{NETWORK MODEL}

The capacity of random wireless networks was first studied in a pioneering seminar work by Gupta and Kumar [7]. There are different approaches to increase the network throughput, such as reducing the interference, the scheduling on the MAC layer, route selection on the routing layer, channel assignment if multi-channels are available, and power control on the physical layer. In this section, we first introduce our network system model, then we discuss in detail the interference models we will use and then define the problem that we will study in this paper.

We consider large scale random networks. Typically there are three ways to increase the number of network nodes to infinity.

1. One is to fix the deployment region and then increase the node density to infinity. This is typically called the dense model. This model is widely studied, e.g., Gupta and $\mathrm{Ku}-$ mar studied the critical transmission range (CTR) [8] and the capacity for unicast [7] using this model.

2. Another way is to fix the node density to a given constant and increase the deployment region to infinity. This is typically called the extended model. Notice that to get a connected network with high probability, we also need to increase the transmission range of nodes. This model is also used by several papers to study the CTR or capacity, e.g., [17,25].

3. The third way is to fix the transmission range of all nodes to some constant, then increase the node density (asymptotically same as the node degree when the transmission range is fixed) and the deployment area to increase the number of nodes in the network. We call this model the constant-range 
model. Assume that $n$ nodes will be deployed. It has been proved in [24] that the minimum node degree for connectivity is $\Theta(\log n)$. This implies that the area of the deployment region is at most $\Theta\left(\frac{n}{\log n}\right)$.

In this paper, we will adopt the third model. Notice that our results presented in this paper actually are immaterial to the model used. Most results presented in this paper rely on the ratio $\frac{a}{r}$ where $a$ is the side-length of the deployment square and $r$ is the transmission range, where either $a$ or $r$ or both could be a function of $n$.

In this paper, we assume that there is a set $V=\left\{v_{1}, v_{2}, \cdots, v_{n}\right\}$ of $n$ communication terminals deployed in a region $\Omega$. We mainly focus the scenario when $\Omega$ is a square with side length $a$. Every wireless node has a uniform transmission range $r$ such that a node $u$ can successfully receive the signal sent by node $v$ if and only if $\|u-v\| \leq r$. The complete communication graph is a undirected graph $G=(V, E)$, where $E$ is the set of communication links.

To schedule two links at the same time slot, we must ensure that the schedule will avoid interference. Several different interference models have been used to model the interferences in wireless networks. In this paper, we will mainly focus on the protocol interference model. We assume that each node $v_{i}$ has a constant interference range $R$. Here any node $v_{j}$ will be interfered by the signal from $v_{k}$ if $\left\|v_{k}-v_{j}\right\| \leq R$ and node $v_{k}$ is sending signal to some node other than $v_{j}$. In this paper, we always assume that the interference range $R$ is within a small constant factor of the transmission range $r$, i.e., $R=\Theta(r)$.

Capacity Definition: We assume that each node $v_{i}$ could serve as the source node for some multicast. For each node $v_{i}$, we randomly select $k-1$ nodes, say $U_{i} \subseteq V-\left\{v_{i}\right\}$, from the remaining $n-1$ nodes as the receivers of multicast session using $v_{i}$ as the source node. Assume that node $v_{i}$ will send data to these receivers $U_{i}$ with a data rate $\lambda_{i}$. Notice that when the receivers are far away from the source node, we need multiple intermediate nodes to relay the data for $v_{i}$. Let $\lambda=\left(\lambda_{1}, \lambda_{2}, \cdots, \lambda_{n-1}, \lambda_{n}\right)$ be the rate vector of the multicast data rate of all multicast sessions. Given a set of $n_{s}$ multicast sessions with the set of source nodes $\mathcal{S} \in V$, let $\lambda_{\mathcal{S}}=\left\{\lambda_{i_{1}}, \lambda_{i_{2}}, \cdots, \lambda_{i_{n_{s}}}\right\}$ be the vector of data rates of all sources in $\mathcal{S}$. When given a fixed network $G=(V, E)$, where the node positions of all nodes $V$, the set of receivers $U_{i}$ for each source node $v_{i}$, and the multicast data rate $\lambda_{i}$ for each source node $v_{i}$ are all fixed, we first define what is a feasible rate vector $\lambda$ for the network $G$.

Definition 1 (FeAsible RATE VECTOR). A multicast rate vector $\lambda=\left(\lambda_{1}, \lambda_{2}, \cdots, \lambda_{n-1}, \lambda_{n}\right)$ (or generally $\lambda_{\mathcal{S}}$ ) bits/sec is feasible if there is a spatial and temporal scheme for scheduling transmissions such that by operating the network in a multi-hop fashion and buffering at intermediate nodes when awaiting transmission, every node $v_{i}$ can send $\lambda_{i}$ bits/sec average to its chosen $k-1$ destination nodes. That is, there is a $T<\infty$ such that in every time interval (with unit seconds) $[(i-1) \cdot T, i \cdot T]$, every node can send $T \cdot \lambda_{i}$ bits to its corresponding $k-1$ receivers.

Given a set $\mathcal{S}$ of $n_{s}$ multicast sessions, the total throughput capacity of such feasible rate vector $\lambda_{\mathcal{S}}$ for multicast is defined as $\Lambda_{k, \mathcal{S}}(n)=\sum_{v_{i} \in \mathcal{S}} \lambda_{i}$. The per node multicast throughput is defined as $\lambda_{k, \mathcal{S}}(n)=\frac{\sum_{v_{i} \in \mathcal{S}} \lambda_{i}}{n_{s}}$, where $k$ is the total number of nodes in each multicast session, including the source node. When $\mathcal{S}$ is clear from context, we write $\Lambda_{k, \mathcal{S}}(n)$ and $\lambda_{k, \mathcal{S}}(n)$ as $\Lambda_{k}(n)$ and $\lambda_{k}(n)$.

DEFINITION 2 (ThroughPUT CAPACITY). A total multicast throughput $\Lambda_{k}(n)$ is feasible for multicast if there is a rate vector $\lambda=\left(\lambda_{1}, \lambda_{2}, \cdots, \lambda_{n-1}, \lambda_{n}\right)$ that is feasible and $\Lambda_{k}(n)=$ $\sum_{i=1}^{n} \lambda_{i}$. Given $n_{s}$ sources $\mathcal{S}$, a per node multicast throughput $\lambda_{k, \mathcal{S}}(n)$ bits/sec is feasible if there is a $\lambda_{\mathcal{S}}=\left(\lambda_{i_{1}}, \lambda_{i_{2}}, \cdots, \lambda_{i_{n_{s}-1}}, \lambda_{n_{s}}\right)$ that is feasible and $\lambda_{k, \mathcal{S}}(n)=\frac{\sum_{i=1}^{n} \lambda_{i}}{n_{s}}$.

Definition 3 (CAPACITY OF RANDom Networks). The total multicast capacity of a class of random networks is of order $\Theta(g(n))$ bits/sec if there are deterministic constants $c>0$ and $c<c^{\prime}<+\infty$ such that

$$
\begin{aligned}
\lim _{n \rightarrow \infty} \boldsymbol{P r}\left(\Lambda_{k}(n)=c g(n) \text { is feasible }\right) & =1 \\
\liminf _{n \rightarrow \infty} \boldsymbol{P r}\left(\Lambda_{k}(n)=c^{\prime} g(n) \text { is feasible }\right) & <1
\end{aligned}
$$

Given $n_{s}$ random sources, we say that the multicast capacity per node of a class of random networks is of order $\Theta(f(n))$ bits/sec if there are deterministic constants $c>0$ and $c<c^{\prime}<+\infty$ such that

$$
\begin{aligned}
\lim _{n \rightarrow \infty} \boldsymbol{P r}\left(\lambda_{k}(n)=c f(n) \text { is feasible }\right) & =1 \\
\liminf _{n \rightarrow \infty} \boldsymbol{P} \boldsymbol{r}\left(\lambda_{k}(n)=c^{\prime} f(n) \text { is feasible }\right) & <1
\end{aligned}
$$

Here the probability is taken for all instances of the random networks $G$ and all set of sources $\mathcal{S}$ with cardinality $n_{s}$.

Useful Known Results: Throughput this paper, we will repeatedly use the following results from probability theory literature.

Lemma 1 (ChebysheV's Inequality). For a variable $X$,

$$
\boldsymbol{P r}(|X-\mu| \geq A) \leq \frac{\operatorname{Var}(X)}{A^{2}}
$$

where $\mu=E(X), \operatorname{Var}(X)$ is the variance of $X$, and $A>0$.

LEMMA 2 (LAW OF LARGE NUMBERS). Consider $n$ uncorrelated variables $X_{i}, 1 \leq i \leq n$ with same expected value $\mu=$ $E\left(X_{i}\right)$ and variance $\sigma^{2}=\operatorname{Var}\left(X_{i}\right)$. Let $\bar{X}=\frac{\sum_{i=1}^{n} X_{i}}{n} . \forall \epsilon>0$,

$$
\operatorname{Pr}(|\bar{X}-\mu|<\epsilon) \geq 1-\frac{\sigma^{2}}{n \cdot \epsilon^{2}}
$$

Lemma 3 (BInomial Distribution). Considern independent variables $X_{i} \in\{0,1\}, p=\boldsymbol{P r}\left(X_{i}=1\right)$, and $X=\sum_{i=1}^{n} X_{i}$.

$$
\begin{aligned}
& \boldsymbol{P r}(X \leq \xi) \leq e^{\frac{-2(n \cdot p-\xi)^{2}}{n}}, \quad \text { when } 0<\xi \leq n \cdot p . \\
& \boldsymbol{P r}(X>\xi)<\frac{\xi(1-p)}{(\xi-n \cdot p)^{2}}, \quad \text { when } \xi>n \cdot p .
\end{aligned}
$$

Notations: Throughput this paper, for a continuous region $\Omega$, we use $|\Omega|$ to denote its area; for a discrete set $S$, we use $|S|$ to denote its cardinality; for a tree $T$, we use $\|T\|$ to denote its total Euclidean edge lengths; $x \rightarrow \infty$ denotes that variable $x$ takes value to infinity.

\section{UPPER BOUNDS ON MULTICAST CA- PACITY FOR RANDOM NETWORKS}

\subsection{The upper-bound on $\frac{a}{r}$}

We assume that $n$ wireless nodes $V$ with transmission range $r$ are randomly and uniformly distributed in a square region with side length $a$. We first study the asymptotic bound on $a / r$ such that the resulted network $G=(V, E)$ is connected almost surely, i.e., with probability going to 1 as $n$ goes to infinity. Notice that for a set of nodes, the CTR for connectivity is always the length of the longest 
edge of the Euclidean minimum spanning tree (EMST) of this set of nodes $[8,16,17]$. Consequently, studying the CTR for connectivity is equivalent to studying the longest edge of the EMST of a set $V$ of nodes when $V$ follows a certain distribution such as Poisson distribution or random uniform distribution.

Assume that $n$ points are distributed uniformly at random in the 2-dimensional square with side length $a$. Let $M_{n, a}$ be the random variable denoting the length of the longest edge of EMST built on this set of $n$ nodes. Then a simple scaling of the result proved in [16] shows that, $\forall \beta, \lim _{n \rightarrow \infty} \operatorname{Pr}\left(n \pi \cdot\left(\frac{M_{n, a}}{a}\right)^{2}-\log n \leq \beta\right)=$ $\frac{1}{e^{e^{-\beta}}}$. Thus, with probability $\frac{1}{e^{e^{-\beta}}}$, we know that the longest edge length $M_{n, a}$, of EMST built on $n$ points distributed in a square with side-length $a$, is at most $\sqrt{\frac{\log n+\beta}{n \pi}} \cdot a$. Thus, when $\beta \rightarrow \infty$ and $a \leq \sqrt{\frac{n \pi}{\log n+\beta}} \cdot r$, we know that the longest edge of EMST has length at most $r$ almost surely. Thus, we have

THEOREM 4. Assume that n nodes, each with transmission range $r$, are randomly uniformly deployed in a square region of side length $a$. When $\frac{a}{r} \leq \sqrt{\frac{n \pi}{\log n+\beta}}$ for $\beta \rightarrow \infty$, the resulted network $G=(V, E)$ is connected with probability at least $\frac{1}{e^{e^{-\beta}}}$.

For example, we can set $a=r \sqrt{\frac{n}{\log n}}$ where $\beta=(\pi-1) \log n$.

\subsection{General Techniques}

In previous studies $[7,18]$ of capacity of random networks, a common approach is to analyze the expected number of hops $H(b)$ a bit $b$ has to travel and the total number of simultaneous transmissions $S=O\left(\frac{a^{2}}{r^{2}}\right)$ possible in the system. If each source node generates data at rate $\lambda$, the number of bits generated by these $n_{s}$ sources in time interval $T$ is simply $\lambda T n_{s}$. Thus, the total number of transmissions of all bits to their destinations is $\lambda T n_{s} H(b)$ almost surely. Consequently, we have $\lambda T n_{s} H(b) \leq T \cdot S$. This implies that $\lambda=O\left(\frac{a^{2}}{r^{2}} \cdot \frac{1}{n_{s} H(b)}\right)=O\left(\frac{n}{\log n} \cdot \frac{1}{n_{s} H(b)}\right)$. In [7], for unicast, Gupta and Kumar essentially used $\Theta\left(\frac{1}{r}\right)$ (assumed $a=1$ ) as estimation of $H(b)$ and derived $\Theta\left(\frac{W}{n_{s} \cdot r}\right)$ as per-node capacity upper-bound. In [18], for multicast, Shakkottai et al. essentially used $H(b)=\Theta\left(\frac{\sqrt{k}}{r}\right)$ (assumed $a=1$ ) to derive $O\left(\frac{W}{n_{s} \cdot \sqrt{k} \cdot r}\right)=$ $O\left(\frac{\sqrt{n}}{n_{s} \sqrt{k \log n}}\right)$ as per-node capacity upper-bound. Although this traditional technique is valid and convenient for studying the asymptotic unicast capacity and the multicast capacity with some special configurations ( $k=n^{1-\epsilon}$ for some $0<\epsilon<1$ ) [18], this may produce a pessimistic or even erroneous upper-bound for asymptotic multicast capacity in a general setting studied in this paper. For example, when $k=n$ (i.e., broadcast), formula $O\left(\frac{\sqrt{n}}{n_{s} \sqrt{k \log n}}\right)$ only produces an upper-bound $O\left(\frac{1}{n_{s} \sqrt{\log n}}\right)$, which is asymptotically smaller than the achievable per-node broadcast capacity $\Theta\left(\frac{1}{n_{s}}\right)$ implied in $[10,11,20]$. The reason for this discrepancy is that for a multicast tree $T$ with total length $\|T\|$, value $\Theta\left(\frac{\|T\|}{r}\right)$ may not give the lower bound on the number of transmissions needed by the tree $T$ due to the multicast natural of wireless transmissions. For the example of broadcast, later we will show that for any broadcast tree $T,\|T\|=\Omega(\sqrt{n})$ almost surely. Thus, $\Theta\left(\frac{\|T\|}{r}\right)=\Omega\left(\frac{n}{\sqrt{\log n}}\right)$ due to $r=\Theta\left(\sqrt{\frac{\log n}{n}}\right)$. On the other hand, a simple broadcast based on a connected dominating set [11] will only require $\Theta\left(\frac{n}{\log n}\right)$ transmissions.

To address the above challenges and discrepancies, we use two new approaches to analyze the upper-bound of multicast capacity:
1. Area Argument: This is based on the area covered by the transmission disks of all internal nodes in a multicast tree;

2. Data Copies Argument: This approach is based on the number of nodes that receive a copy of a multicast data during the transmissions of all nodes in the tree.

The area argument essentially works as follows. When we multicast from one source node $v_{i}$ to all its $k-1$ receivers $U_{i}$, all nodes lying inside the interference region of any transmitting node for this multicast session cannot receive data from other nodes simultaneously. For any node $u$, let $t_{i}(u)$ be the time-intervals that node $u$ will transmit data for multicast tree $T_{i}$. Thus, a multicast tree will claim a number of cylinders $\left(D(u, r) \times t_{i}(u)\right.$ for internal node $u$ in $T$ ) in the space-time dimension $\mathcal{R}^{2} \times \mathcal{T}$, where $D(u, r)$ denotes the transmission disk of node $u, \mathcal{T}$ is the scheduling period. Thus, given a multicast tree $T_{i}$ for multicast originated from $v_{i}$, the pairs of $\left(D(u, r), t_{i}(u)\right)$ (i.e., transmission disk $D(u, r)$ will be used for multicast originated at $v_{i}$ during the transmission time-interval $t_{i}(u)$ ) claimed by this multicast should be disjoint from the pairs claimed by other multicast sessions. Thus, it is not difficult to prove the following lemma:

Lemma 5. For any operation $\mathcal{O}$, such as multicast, let $\mathcal{A}$ be the area of the region defined by uniting the transmission regions of all transmitting nodes. If $\mathcal{A}$ is at least $\wp$ with high probability, then, w.h.p., the aggregated capacity for this operation in a random network deployed in a region with area $\Phi$ is at most $\frac{\Phi \cdot W}{\wp}$.

The data-copies argument works as follows. When we multicast from one source node $v_{i}$ to all its $k-1$ receivers $U_{i}$, it is more likely that other nodes will also get a copy of the data. Here, for the purpose of analysis, when a node $v$ sends data to one of its neighboring nodes, all its neighboring nodes will be charged a copy of the data. Notice that here a neighboring node $w$ may not be the intended receiver. However, since when $v$ is transmitting, any of its neighboring node $w$ cannot receive data simultaneously from any other transmitting node due to interference, we will say that node $w$ also gets a copy of the data. For multicast with $k-1$ receivers, clearly, at least $k$ nodes will get a copy of the data. Generally, assume that $C_{i}$ nodes will get a copy of the data when the $k-1$ receivers are randomly selected for each possible source node $v_{i}$. Obviously, $\sum_{v_{i} \in \mathcal{S}} \lambda_{i} \cdot C_{i} \leq n \cdot W$. Further assume that $C_{i} \geq C$ almost surely, i.e., $\operatorname{Pr}\left(C_{i} \geq C\right) \rightarrow 1$ as $n$ or $k$ goes to infinity. Then the total multicast capacity satisfies, almost surely,

$$
\Lambda_{k}(n)=\sum_{v_{i} \in \mathcal{S}} \lambda_{i} \leq \frac{n \cdot W}{C} .
$$

Clearly, $C \geq k$. Next subsection is devoted to give a better lower bound on $C$. The following lemma is straightforward.

LEMMA 6. For any operation $\mathcal{O}$, such as multicast, let $\mathcal{X}$ be the number of nodes that will receive $a$ copy of the data (i.e., fall inside the interference region of any one of its transmitting nodes). Assume that $\mathcal{X}$ is at least $\mathcal{N}$ with high probability. Then, with high probability, the aggregated capacity for this operation by all nodes in a random network of $n$ nodes is at most $\frac{n \cdot W}{\mathcal{N}}$.

In our proofs, we will utilize these two technical lemmas to give upper-bound on the capacity of a random network for an operation that will be performed by each node of the network, such as multicast. Notice that the above lemmas require us to find largest $\wp$ such that $\operatorname{Pr}(\mathcal{A} \geq \wp) \rightarrow 1$, or the largest $\mathcal{N}$ such that $\operatorname{Pr}(\mathcal{X} \geq \mathcal{N}) \rightarrow$ 1 . In some cases, such $\wp$ may be much smaller than the mean value $E(\mathcal{A})$ of $\mathcal{A}$; such $\mathcal{N}$ may be much smaller than the mean value 
$E(\mathcal{X})$ of $\mathcal{X}$. In these cases, we could rely on a much stronger technical lemmas based on law of large numbers when the number $n_{s}$ of operations needed to perform goes to infinity. For example,

LEMMA 7. For $n_{s}=f(n)$ multicast sessions $\left\{\mathcal{O}_{i} \mid 1 \leq\right.$ $\left.i \leq n_{s}\right\}$, where $\mathcal{O}_{i}$ has node $v_{i}$ as source, let $\mathcal{X}_{i}$ be the number of nodes that will receive $a$ copy of the data sent by some transmitting nodes in $\mathcal{O}_{i}$. Assume that variables $\mathcal{X}_{i}$ are independent, and $E\left(\mathcal{X}_{i}\right)=\mathcal{N}$ for all $i$. If $\lim _{n \rightarrow \infty} \frac{\sigma^{2}}{n_{s}}=0$ then, w.h.p., the pernode multicast capacity for this operation in a random network of $n$ nodes is at most $O\left(\frac{n \cdot W}{n_{s} \mathcal{N}}\right)$.

Proof. Let $\lambda$ be the per-node capacity achievable. Then $\lambda$. $\sum_{i=1}^{n_{s}} \mathcal{X}_{i} \leq n \cdot W$. Let $\bar{X}=\frac{\sum_{i=1}^{n_{s}} \mathcal{X}_{i}}{n_{s}}$ and $\sigma^{2}=\operatorname{Var}\left(\mathcal{X}_{i}\right)$. Then the law of large numbers (Lemma 2 ) implies that, for any $\epsilon>0$, $\operatorname{Pr}(|\bar{X}-\mathcal{N}|<\epsilon) \geq 1-\frac{\sigma^{2}}{n_{s} \cdot \epsilon^{2}}$. Thus, as long as $\lim _{n \rightarrow \infty} \frac{\sigma^{2}}{n_{s}}=$ 0 , we have $\bar{X} \geq \mathcal{N}-\epsilon$ almost surely. Thus, we have $\lambda \leq$ $\frac{n \cdot W}{n_{s}(\mathcal{N}-\epsilon)}=O\left(\frac{n \cdot W}{n_{s} \mathcal{N}}\right)$ almost surely.

To our surprise, we find that the multicast capacity of a random network where each multicast session has $k-1$ receivers has two regimes: when the number of receivers $k-1$ is over some threshold, multicast capacity is asymptotically same as the broadcast capacity; otherwise, the multicast capacity decreases linearly over $\frac{1}{\sqrt{k}}$. Next, we will provide upper-bounds for each case separately.

\subsection{When $k=O\left(a^{2} / r^{2}\right)$}

We first study the multicast capacity when the number of receivers is at most $\Theta\left(a^{2} / r^{2}\right)$. We will present upper bound of the total multicast capacity. A trivial upper bound for total multicast capacity is $W \cdot n$ since there are $n$ source nodes and each source node can only send $W$ bits/sec. A refined upper bound is $\frac{n \cdot W}{k}$ which is derived from the perspective of recipients: (1) each node can receive at most $W$ bits/sec, and (2) among received data by all nodes, any data from any source node will have at least $k$ copies (one copy at each of the $k-1$ receivers and one copy at the source node). From Lemma 6 , for a multicast tree $T_{i}$ spanning source node $v_{i}$ and $k-1$ receivers $U_{i}$, we would like to know the (expected) number of internal nodes used in $T_{i}$. To analyze this value, we first study the asymptotic lower bound of the Euclidean length $\left\|T_{i}\right\|$ of a multicast tree $T_{i}$.

LEMMA 8. [4] Given any $k$ nodes $U$, any multicast tree spanning these $k$ nodes (may be using some additional relay nodes) will have an Euclidean length at least $\varrho \cdot\|E M S T(U)\|$, where $\varrho \geq \frac{\sqrt{3}}{2}$ and $\operatorname{EMST}(U)$ is the EMST spanning $U$.

Observe that the tight bound on $\varrho=\frac{\sqrt{3}}{2}$ is the famous Gilbert and Pollak conjecture which was proved by Du and Hwang in 1992 [4]. A bound $\varrho \geq \frac{1}{2}$ can be easily proved as follows. For any steiner multicast tree $T$ spanning these $k$ nodes, we construct an Euler tour on this tree. Clearly the total length of the Euler tour (EC) is 2 times of the length of the multicast tree $T$. On the other hand, the Euler tour has length at least that of the Euclidean minimum spanning tree for these $k$ nodes. The statement follows from $\|E M S T\|<$ $\|E C\|=2 \cdot\|T\|$. Recall that in this paper, $\|T\|$ denotes the total Euclidean length of all links in a structure $T$.

Based on Lemma 8, to get a lower bound on $\left\|T_{i}\right\|$ of any multicast tree $T_{i}$, we need study the length of EMST spanning these $k$ random nodes. In [19], Steele established the following result:

LEMMA 9. The total edge length of the EMST of $n$ nodes randomly and uniformly distributed in a d-dimensional cube of sidelength a is asymptotic to $\tau(d) \cdot n^{\frac{d-1}{d}} \cdot a$, where $\tau(d)$ is a constant depending only on the dimension $d$.
Thus, based on Lemma 8 and Lemma 9, we have

LeMma 10. The total edge length, denoted by $\left\|T_{i}\right\|$, of any multicast tree $T_{i}$ spanning $k$ nodes randomly placed in a square of side-length a almost surely is at least $\varrho \cdot \tau(2) \cdot \sqrt{k} \cdot a$, when $k \rightarrow \infty$.

From now on, for simplicity, we will denote $\tau \leftarrow \sqrt{3} \tau(2) / 2$. When the number of receivers does not go to infinity when $n \rightarrow \infty$, we can use the law of large numbers to show that the expected value of $n_{s}$ multicast tree lengths $\left\|T_{i}\right\|$, for $v_{i} \in \mathcal{S}$ is at least $\tau \cdot \sqrt{k} \cdot a$ almost surely. Let $X=\|E M S T(U)\|$ of a set of $k$ randomly selected nodes $U$ in a square of side-length $a$. It was shown in [19] that $\operatorname{Var}(X) \ll a^{2} \cdot \log k$. We then show that $X \leq 2 \sqrt{2} \sqrt{k} a$.

LEMMA 11. For any $k$ nodes $U$ placed in a square region with side-length $a$, the length of EMST spanning $U$ is at most $2 \sqrt{2} \sqrt{k} a$.

Proof. Given $k$ nodes in the square, we will use Prim's algorithm to construct EMST: originally each node is a component, and then we iteratively find a shortest edge to connect two components to form a larger component until only one component is left. Consider the $(k+1-g)$-th step (for $1 \leq g \leq k$ ), which has $j$ connected components as input. For $g \geq 2$, if we partition the square into a $\lfloor\sqrt{g-1}\rfloor$ by $\lfloor\sqrt{g-1}\rfloor$ grid with side-length $\frac{a}{\lfloor\sqrt{g-1}\rfloor}$, then there is at least one cell that contains at least two connected components. This implies that the shortest edge connecting components at the $(k+1-g)$ th step is at most $\sqrt{2} \frac{a}{\lfloor\sqrt{g-1}\rfloor}$. Consequently, the EMST has length at most $\sum_{j=2}^{k} \sqrt{2} \frac{a}{\lfloor\sqrt{g-1}\rfloor} \leq$ $\sum_{i=1}^{\sqrt{k-1}} \sqrt{2} \frac{a \cdot\left((i+1)^{2}-i^{2}-1\right)}{i} \leq 2 \sqrt{2} \sqrt{k-1} \cdot a$.

Bound the data copies: A straightforward lower-bound on the expected number of nodes (including leaf nodes) needed in a multicast tree spanning $k$ nodes randomly selected in a square of sidelength $a$ is $\tau \cdot \sqrt{k} \cdot \frac{a}{r}$ with high probability. This bound can be derived as follows: (1) the expected Euclidean length of a multicast tree is at least $\tau \cdot \sqrt{k} \cdot a$, and (2) the transmission range of each node is only $r$, thus, removing one tree edge incident on a leaf node will reduce the total edge length by at most $r$ and we will reduce the number of nodes by 1 . Consequently, we have $C \geq \tau \cdot \sqrt{k} \cdot a / r$. Although this bound on $C$ is much better than bound $C \geq k$ when $k=O\left(a^{2} / r^{2}\right)$, the bound can be further improved based on the following observation. When nodes on the multicast tree relay data from the source node to receivers, not only its downstream nodes of the multicast tree will receive the data, but also all its neighboring nodes (in communication graph $G$ ) will get a copy of the data. We will then analyze the number of nodes that will get the copy of the data. Given a multicast tree $T$, let $D(T)$ be the region covered by all transmitting disks of all transmitting nodes (internal nodes) in the multicast tree $T$. Observe that the leaf nodes do not contribute to $D(T)$ at all here. See Figure 1 (a) for illustration. Clearly, the area of $D(T)$, denoted by $|D(T)|$, is at most $|D(T)| \leq 2 r \cdot\|T\|$ where $\|T\|$ is the total Euclidean length of all links in $T$. We will prove that the area of $D(T)$ is also at least $\frac{\tau \sqrt{k} a \cdot r}{c_{0}}$ for some constant $c_{0}$ independent of the network.

LEMMA 12. The area of the region $D(T)$, denoted by $|D(T)|$ is at most $\tau \sqrt{k} a \cdot 2 r$ and w.h.p.is at least $\frac{\tau \sqrt{k} a \cdot r}{c_{0}}$ when $k<\left(\frac{\tau(1-(6(d+1) \cdot \rho))}{6(d+1)+1}\right)^{2}$. $\frac{a^{2}}{r^{2}}$, for some constant $c_{0}=1 /(\rho \pi)$, where $0<\rho<\frac{1}{6(d+1)}$ and constant $d \leq 13$.

PROOF. For any multicast tree $T$ spanning source node $v_{i}$ and the set of receivers $U_{i}$, for convenience, let $V(T)$ be the set of nodes in tree $T$; let $U_{i}^{\prime}=U_{i} \cup\left\{v_{i}\right\}$; let $I(T)$ be all the Steiner 


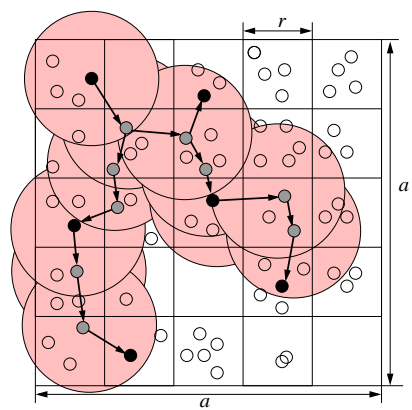

(a) Region $D(T)$

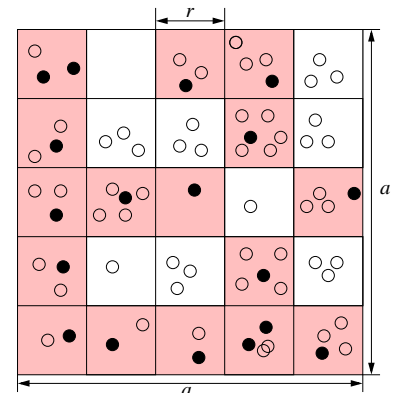

(b) Dense receivers
Figure 1: (a) Region $D(T)$ covered by transmitting disks of internal nodes in multicast tree $T$. Here the solid black nodes are receivers/source and gray nodes are Steiner nodes. (b) Partition of square with side-length $a$ into squarelets with side-length $r$. Here the solid black nodes are receivers/source. Shaded squarelets are squarelets with at least one receiver.

nodes used to connect them, i.e., $I(T)=V(T) \backslash U_{i}^{\prime}$. Clearly the communication graph defined on $V(T)$ (where two nodes are connected iff their Euclidean distance is no more than $r$ ) is connected. We use $G_{T}$ to denote such induced graph. We will then build another multicast tree $T^{\prime}$ from $G_{T}$ to connect nodes $U_{i}^{\prime}$.

In graph $G_{T}$, we build a connected dominating set (CDS) using a method described in $[3,21]$. Source node $s$ will be added to the CDS if it is not in the CDS. It has been proved in $[3,21]$ that, in the constructed CDS, each node on the CDS has a degree bounded by a constant, say $d$. For example, it can be shown that the degree of node in CDS is bounded by 13 if the method presented in [21] is used. The multicast tree $T^{\prime}$ is then a simple breadth-first-search tree computed from the CDS, rooted at the source node $s$.

We essentially will prove that each point from the region $D\left(T^{\prime}\right)$ is covered by at most a constant $c_{0}$ number of disks from the multicast tree $T^{\prime}$. For each point $p$ in the region, we divide the disk $D(p, r)$ centered at point $p$ with radius $r$ into 6 equal sized sectors. Thus, any pair of nodes falling into the same sector will be within distance of $r$ of each other, and thus connected in the original communication graph. Consequently, for each point $p$, the number of disks from $D\left(T^{\prime}\right)$ that cover $p$ is at most $6(d+1)$. If it is at least $6(d+1)+1$, then at least one of the sectors will have at least $d+2$ nodes, which implies that any node in that sector will have degree at least $d+1$ in the induced CDS graph. This is a contradiction to the fact that the degree of induced CDS is bounded by $d$. Thus, the area of the region $D\left(T^{\prime}\right)$ is thus at least $\frac{\left|I\left(T^{\prime}\right)\right| \cdot \pi r^{2}}{6(d+1)}$, where $d$ is the degree bound on the induced CDS graph constructed. Here $\left|I\left(T^{\prime}\right)\right|$ is the number of internal nodes in multicast tree $T^{\prime}$.

Notice that some leaf nodes in $T$ may become internal nodes in $T^{\prime}$; some internal nodes may not be used by tree $T^{\prime}$ at all. Let $A(T)$ be the region covered by all disks centered at all nodes of a tree $T$, including the leaf nodes. Let $\ell(T)$ be the number of leaf nodes in a tree $T$. Obviously, $|D(T)|+\ell(T) \cdot \pi r^{2} \geq|A(T)| \geq\left|A\left(T^{\prime}\right)\right| \geq$ $\left|D\left(T^{\prime}\right)\right|$. Thus,

$$
|D(T)| \geq\left|D\left(T^{\prime}\right)\right|-\ell(T) \cdot \pi r^{2} \geq \frac{\left|I\left(T^{\prime}\right)\right| \cdot \pi r^{2}}{6(d+1)}-\ell(T) \cdot \pi r^{2} .
$$

Obviously, $\ell(T) \leq k$. For a multicast tree $T^{\prime}$, there are at most $k$ leaf nodes. If we remove all edges in $T^{\prime}$ incident on leaf nodes, the total edge length of all edges left is at least $\left|T^{\prime}\right|-k \cdot r$. Thus, the number of internal nodes $\left|I\left(T^{\prime}\right)\right|$ in $T^{\prime}$ is at least $\frac{\left|T^{\prime}\right|-k \cdot r}{r}$. Notice that $T^{\prime}$ is a tree spanning the source node $s$ and all receivers $U_{i}^{\prime}$. Thus, with high probability, $\left|T^{\prime}\right| \geq \tau \sqrt{k} \cdot a$ since $U_{i}^{\prime}$ has $k$ nodes. Thus, with high probability, we have $\left|I\left(T^{\prime}\right)\right| \geq \frac{\tau \sqrt{k} \cdot a}{r}-k$. Assume that $k<\left(\frac{\tau(1-(6(d+1) \cdot \rho))}{6(d+1)+1}\right)^{2} \cdot \frac{a^{2}}{r^{2}}$, which implies $|D(T)|$ is at least $\frac{\left|I\left(T^{\prime}\right)\right| \cdot \pi r^{2}}{6(d+1)}-\ell(T) \cdot \pi r^{2} \geq\left(\frac{\frac{\tau \sqrt{k} \cdot a}{r}-k}{6(d+1)}-k\right) \cdot \pi r^{2} \geq \rho \pi \tau \sqrt{k} \cdot a \cdot r$. For example, we can set $\rho=\frac{1}{12(d+1)}$. This finishes the proof.

For convenience, hereafter, we use

$$
\theta_{1}=\left(\frac{\tau(1-(6(d+1) \cdot \rho))}{6(d+1)+1}\right)^{2}
$$

to denote the threshold value such that Lemma 12 is true if $k<$ $\theta_{1} \cdot \frac{a^{2}}{r^{2}}$. Based on Lemma 12, we know that the expected number, denoted by $C$, of nodes from $V$ that is in the region $D(T)$ is at least

$$
|D(T)| \cdot \frac{n}{a^{2}} \geq \frac{\tau \cdot \sqrt{k} \cdot a \cdot r \cdot n}{c_{0} a^{2}}=\frac{\tau \cdot \sqrt{k} \cdot r \cdot n}{c_{0} a}
$$

Recall that we assume that there is only one single channel in the network. It is then not difficult to show the following lemma:

LEMMA 13. With high probability, the number $C$ of nodes that get a copy of the multicast data satisfies $C>\frac{\tau \cdot r \cdot \sqrt{k} \cdot n}{2 c_{0} a}$.

Proof. Consider a multicast tree $T$. Notice that $n$ wireless nodes will be randomly distributed in a square region of side-length $a$. Let $X_{i}=\{0,1\}$ be an indicator variable whether the $i$ th node $v_{i}$ will fall inside the region $D(T)$ for a multicast tree $T$. Clearly $\operatorname{Pr}\left(X_{i}=1\right)=\frac{|D(T)|}{a^{2}}$. Recall that, we already proved that, with high probability, $|D(T)| \geq \frac{\tau \sqrt{k} a \cdot r}{c_{0}}$. Thus, we have $\operatorname{Pr}\left(X_{i}=1\right) \geq$ $\frac{\tau \sqrt{k} \cdot r}{c_{0} \cdot a}$. Obviously, $X=\sum_{i=1}^{n} X_{i}$ is the expected number of nodes falling inside the region $D(T)$, which is also the number $C$ of nodes that will get a copy of the data by multicast. Then the expected value $E(X) \geq \frac{\tau \sqrt{k} \cdot r \cdot n}{c_{0} \cdot a}$. Based on Lemma 3, we have $\operatorname{Pr}\left(C \leq n \cdot \frac{|D(T)|}{2 a^{2}}\right) \leq e^{\frac{-2\left(n \cdot \frac{|D(T)|}{a^{2}}-n \cdot \frac{|D(T)|}{2 a^{2}}\right)^{2}}{n}}=e^{\frac{-n \cdot|D(T)|^{2}}{2 a^{4}}}$

Notice that to guarantee a connected network with high probability, we have $a<\sqrt{\frac{\pi n}{\log n}} \cdot r$ with high probability. Thus,

$\operatorname{Pr}\left(C \leq n \cdot \frac{|D(T)|}{2 a^{2}}\right) \leq e^{-\frac{n \cdot \tau^{2} \cdot k \cdot r^{2}}{2\left(c_{0}\right)^{2} \cdot a^{2}}} \leq e^{-\frac{\tau^{2} \cdot k \cdot \log n}{2 \pi c_{0}^{2}}}=\frac{1}{n^{\frac{\tau^{2} \cdot k}{2 \pi c_{0}^{2}}}}$

Consequently, when $n \rightarrow \infty, \operatorname{Pr}\left(C \leq n \cdot \frac{|D(T)|}{2 a^{2}}\right) \rightarrow 0$. Thus,

$$
\operatorname{Pr}\left(C>\frac{\tau \cdot r \cdot \sqrt{k} \cdot n}{2 c_{0} \cdot a}\right) \geq \operatorname{Pr}\left(C>n \cdot \frac{|D(T)|}{2 a^{2}}\right) \rightarrow 1 .
$$

This finishes the proof.

Consequently, we have the following theorem:

THEOREM 14. The multicast capacity with $k-1$ receivers for $n$ nodes that are randomly and uniformly deployed in a square with side-length $a$ is at most $c_{1} \cdot \frac{a W}{r \sqrt{k}}$ for some constant $c_{1}$ when $k<$ $\theta_{1} \cdot a^{2} / r^{2}$.

PROOF. Notice that the multicast capacity is at most $\frac{n W}{C}$ and, with high probability, $C \geq \frac{\tau \cdot r \cdot \sqrt{k} \cdot n}{2 c_{0} a}$ when $k<\theta_{1} \cdot a^{2} / r^{2}$. Thus, the multicast capacity $\Lambda_{k}(n)$ is at most $\frac{n W \cdot 2 c_{0} a}{\tau \cdot r \cdot \sqrt{k} \cdot n}=c_{1} \cdot \frac{a W}{r \sqrt{k}}$ for a constant $c_{1}=\frac{2 c_{0}}{\tau}$. This finishes the proof. 
Recall that we have proved that, to guarantee that we have a connected network with high probability, we need $a \leq r \sqrt{\frac{n \pi}{\log n+\beta}}$ for $\beta \rightarrow \infty$. Thus, letting $c_{2}=c_{1} \sqrt{\pi}$, we have the following theory:

THEOREM 15. The multicast capacity for a random network of $n$ nodes, when $k<\theta_{1} \cdot a^{2} / r^{2}=O\left(\frac{n}{\log n}\right)$, is at most

$$
\Lambda_{k}(n) \leq c_{2} \cdot \frac{\sqrt{n}}{\sqrt{\log n} \cdot \sqrt{k}} \cdot W=O\left(\frac{\sqrt{n}}{\sqrt{\log n} \cdot \sqrt{k}} \cdot W\right) .
$$

With $n_{s}$ multicast sessions, the per node multicast capacity is

$$
\lambda_{k}(n)=\min \left(W, \frac{\Lambda_{k}(n)}{n_{s}}\right)=O\left(\min \left(W, \frac{\sqrt{n}}{n_{s} \sqrt{\log n} \cdot \sqrt{k}} \cdot W\right)\right) .
$$

Notice that Theorem 14 was proved under the assumption that $k \rightarrow \infty$. When this is not the case, we can prove that the per-node multicast capacity $\lambda$ (when each source node generates multicast data at rate $\lambda$ ) also satisfies that $\lambda_{k}(n)=O\left(\frac{\sqrt{n}}{n_{s} \sqrt{\log n} \cdot \sqrt{k}} \cdot W\right)$ when $n_{s} \rightarrow \infty$. Since $k$ is constant in this case, we know that the pernode multicast capacity is upper-bounded by the per-node unicast capacity with $n_{s}$ unicast sessions. Thus, the per-node multicast capacity is almost surely at most $O\left(\frac{n}{n_{s} \sqrt{\log n}} \cdot W\right)$, which is same as $O\left(\frac{n}{n_{s} \sqrt{\log n} \cdot \sqrt{k}} \cdot W\right)$ since $k$ is constant.

\subsection{When $k=\Omega\left(\frac{a^{2}}{r^{2}}\right)$}

In the previous subsection, we showed an upper bound of the multicast capacity when $k<\theta_{1} \cdot a^{2} / r^{2}$. In this subsection we will present an upper bound on multicast capacity when $k \geq \theta_{1} \cdot a^{2} / r^{2}$. We will essentially show that in this case, multicast is asymptotically equivalent to broadcast. Broadcast capacity of single-source of an arbitrary network has been studied in $[11,20]$. In this paper, we will prove that the achievable integrated multicast capacity is only $\Theta(W)$ if an arbitrary $k$ subset of the $n$ nodes will serve as receivers for each possible source node $v_{i}$.

We partition the square of side-length $a$ into squarelets, each with side length $r$. The square will be partitioned into $M=\left\lceil a^{2} / r^{2}\right\rceil$ squarelets, say $B_{1}, B_{2}, \cdots, B_{M}$. Recall that we will randomly select $k \geq \theta_{1} \cdot a^{2} / r^{2}$ receivers in the square region. See Figure 1 (b) for illustration.

LEMMA 16. With high probability, at least $\rho \cdot M$ squarelets will have at least one receiver when $k \geq \theta_{1} \cdot a^{2} / r^{2}$ for a constant $\theta_{1}$.

PROOF. Let $X$ be the number of squarelets that do not have any receivers inside, and $A$ be a fixed fraction of squarelets, say $A=\rho \cdot M$ for a constant $0<\rho<1$. Let $X_{i}$ be indicator variable whether squarelet $B_{i}$ is empty of receivers $\left(X_{i}=1\right.$ is empty). Then $X=\sum_{i=1}^{M} X_{i}$. Notice $\operatorname{Var}(X)=\operatorname{Var}\left(\sum_{i=1}^{M} X_{i}\right)=$ $\sum_{i=1}^{M} \sum_{j=1}^{M} \operatorname{Cov}\left(X_{i}, X_{j}\right)$, where $\operatorname{Cov}\left(X_{i}, X_{j}\right)=E\left(X_{i} \cdot X_{j}\right)-$ $E\left(X_{i}\right) E\left(X_{j}\right)$ is the covariance of variable $X_{i}$ and $X_{j}$. We then compute such $\operatorname{Cov}\left(X_{i}, X_{j}\right)$ for all possible pairs of $i$ and $j: \operatorname{Cov}\left(X_{i}, X_{i}\right)$ $E\left(X_{i}\right)-E\left(X_{i}\right)^{2}$ and $E\left(X_{i}\right)=\left(1-\frac{1}{M}\right)^{k}$; and $E\left(X_{i} \cdot X_{j}\right)=$ $\left(1-\frac{2}{M}\right)^{k}$ if $i \neq j$. Consequently, we have $\operatorname{Var}(X)=M(M-$ 1) $\left[\left(1-\frac{2}{M}\right)^{k}-\left(1-\frac{1}{M}\right)^{2 k}\right]+M\left[\left(1-\frac{1}{M}\right)^{k}-\left(1-\frac{1}{M}\right)^{2 k}\right]$. Since $\left[\left(1-\frac{2}{M}\right)^{k}-\left(1-\frac{2}{M}+\frac{1}{M^{2}}\right)^{k}\right] \leq 0$, we have $\operatorname{Var}(X) \leq$ $M\left[\left(1-\frac{1}{M}\right)^{k}-\left(1-\frac{1}{M}\right)^{2 k}\right]$. From Lemma 1, we have

$$
\operatorname{Pr}(X-E(X) \geq \rho \cdot M) \leq \frac{M\left[\left(1-\frac{1}{M}\right)^{k}-\left(1-\frac{1}{M}\right)^{2 k}\right]}{\rho^{2} M^{2}}
$$

From $k \geq \theta_{1} \cdot M, E(X)=M \cdot\left(1-\frac{1}{M}\right)^{k} \leq M \cdot e^{-\theta_{1}}$. Thus,

$$
\operatorname{Pr}\left(X \geq\left(e^{-\theta_{1}}+\rho\right) \cdot M\right) \leq \frac{\left(\frac{1}{e}\right)^{\theta_{1}}-\left(\frac{1}{e}\right)^{2 \theta_{1}}}{\rho^{2}} \cdot \frac{1}{M}
$$

When $M \rightarrow \infty$, the probability goes to zero. We can also show that, with high probability, there is at most a constant fraction of squarelets that will be empty of receivers. This finishes the proof.

We then prove that the union of the transmission disks of these $k$ nodes in a multicast will cover at least a constant fraction, say $0<\rho_{2} \leq 1$, of the deployment region.

LEMMA 17. The union of the transmission disks of these $k$ nodes $(k-1$ receivers and 1 source node) in a multicast will cover at least a constant fraction, say $0<\rho_{2} \leq 1$, of the deployment region.

PROOF. Based on lemma 16, we know that among $M$ squarelets partitioned from the deployment region, there are at least $\rho \cdot M$ squarelets, each of which contains at least one receiver (or source) node inside. In each such a squarelet $B_{j}$, there is at least one receiver and thus at least one transmitting node in the multicast tree that covers this receiver. The transmitting node must lie inside this squarelet or 8 adjacent squarelets. On the other hand, each transmitting disk can cover receivers from at most 9 squarelets. Consequently, we must have at least $\rho \cdot M / 9$ transmitting disks to cover receivers from $\rho \cdot M$ squarelets. Recall that the squarelet side-length is $r$, which implies that each point in the deployment region is covered by at most 9 such representative transmission disks. Consequently, the total area covered by these representative transmission disks is at least $\rho \cdot M \cdot \pi r^{2} / 81$. Recall that the deployment region has area $a^{2}$ and $M=\left\lceil a^{2} / r^{2}\right\rceil$. Thus, the area of all transmission disks of all these $k$ nodes is at least $\rho_{2}=\frac{\rho \cdot \pi}{81}$ fraction of the total area of the deployment region. This finishes the proof.

Based on Lemma 17 and Lemma 5, we have

THEOREM 18. When $k \geq \theta \cdot a^{2} / r^{2}$ for a constant $\theta$, with high probability, $\Lambda_{k}(n) \leq \frac{W \cdot a^{2}}{\rho_{2} a^{2}}=\frac{W}{\rho_{2}}=O(W)$,x where $\rho_{2}$ is a constant depending only on $\theta$.

Notice that for broadcast, it has been proved in $[11,20]$ that the broadcast capacity is only $\Theta(W)$. Here we essentially prove that for multicast, when the number of receivers is large enough (at least $\left.\Omega\left(\frac{a^{2}}{r^{2}}\right)\right)$, the asymptotic multicast capacity is also only $O(W)$.

\section{LOWER BOUNDS ON MULTICAST CA- PACITY WITH RANDOM NETWORKS}

In this section, we will provide a multicast scheme and prove that the multicast capacity achieved by our scheme matches the asymptotic upper bounds.

\subsection{Good Approximation of MCDS}

Our multicast scheme is based on a good approximation of a minimum connected dominating set (MCDS) of a random network. For each randomly generated network instance, we will first construct an approximation of MCDS. For example, we can use the method introduced in [3] (Algorithm 1 in [3]). This method first finds a maximal independent set using a greedy approach, and then connects them using relay nodes. An maximal independent set (MIS) can be constructed as follows: originally all nodes are marked as white nodes and each node $v$ is assigned a unique $\operatorname{rank}(v)$; a node $v$ is selected to MIS (and marked as black node consequently) if and only if it has the smallest rank among all its white neighbors; Clear such MIS is a dominating set. Then, we connect every pair of dominating nodes that are at most 3 hops away using the least-hop path. The nodes on such least-hop paths will be marked as connectors. The set of all MIS nodes and connectors will form 
a connected dominating set [3]. We then show that we can schedule the transmissions of all nodes in CDS in a constant time-slots without interference; the CDS is a length spanner.

TDMA Scheduling with Constant Slots: For each node on the CDS, there are at most a constant number of neighboring nodes on CDS. In other words, the degree of any node on CDS is bounded by a constant, say $D_{1}$. Using this property, it is easy to show that the number of nodes in the CDS constructed above that can interfere with any node in the CDS is at most a constant. For each node $v$, consider two circles both centered at the node and with radii $R$ and $R+r$. Consider a node $u$ whose transmission will interfere with the transmission of node $v$. Clearly node $u$ will be completely inside the disk centered at $v$ with radius $R+r$. On the other hand we know each circle centered at $u$ with radius $r$ contains at most $D_{1}+1$ nodes. Let $\Delta$ be the maximum number of nodes in CDS whose transmission will interfere with the transmission of a node $v$ in CDS. Using the area argument, we can show that

$$
\Delta \leq \frac{\pi \cdot(R+2 r)^{2} \cdot\left(D_{1}+1\right)}{\pi \cdot r^{2}}=\left(1+\frac{2 R}{r}\right)^{2}\left(D_{1}+1\right) .
$$

This property ensures that we can schedule the transmissions of all nodes in CDS by a TDMA manner such that all nodes will be able to transmit at least once in every $\Delta+1$ time slots. Consequently, the per-node data transmission data rate achieved by nodes on the CDS is at least $\frac{W}{\Delta+1}$. Notice that here $\Delta$ is a constant.

Length Spanner Property: For any two nodes $u$ and $v$ in the network, if $\|u-v\|>r$, then the shortest path connecting $u$ and $v$ via the CDS (constructed above) has length at most 3 times the length of the shortest path connecting them in the original random communication graph $G=(V, E)$ (see Lemma 5 of [3]). Notice that here when $u$ (or $v$ or both) is not in the CDS, we will first connect $u$ (or $v$ or both) to one of its dominators (say $u^{\prime}$ and $v^{\prime}$ ) in the CDS. Then we find the shortest path connecting these corresponding dominators $u^{\prime}$ and $v^{\prime}$ in the CDS.

\subsection{When $k=O\left(a^{2} / r^{2}\right)$}

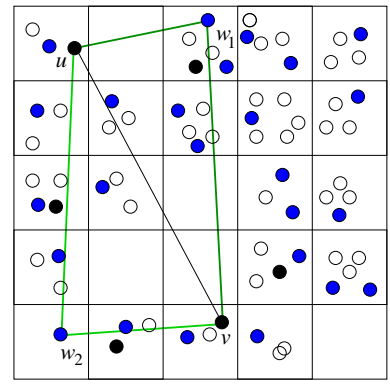

(a) Squarelets partition

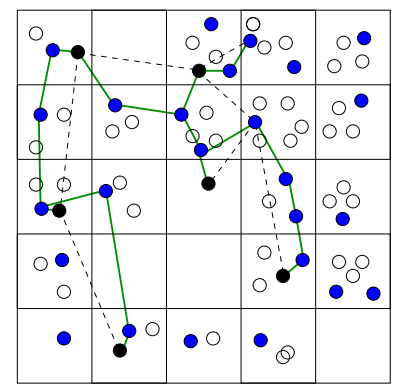

(b) Manhattan Routing Tree
Figure 2: (a) Partition of square with side-length $a$ into squarelets with side-length $r / \sqrt{5}$. For an edge $u v \in$ $E M S T\left(U_{1}^{\prime}\right)$, find a node $w$ (either node $w_{1}$ or node $w_{2}$ which has same row as $u$ and same column as $v$ ) to connect them. (b) a multicast tree constructed using Manhattan approach, where dotted lines denote original EMST of nodes in a multicast session.

When the number of receivers, plus the source node, $k$ is only $O\left(\frac{a^{2}}{r^{2}}\right)$, we will construct a multicast tree from CDS. Consider an instance of a random network $G=(V, E)$ and also an instance of multicast with $v_{1}$ as the source node and $U_{1}=\left\{v_{2}, v_{3}, \cdots v_{k}\right\}$ as the receiver nodes. Let $U_{1}^{\prime}=\left\{v_{1}, v_{2}, v_{3}, \cdots v_{k}\right\}$. We will construct a multicast structure as follows:

$\overline{\text { Algorithm } 1 \text { Multicast Capacity Achieving Manhattan Routing }}$ Based on a Squarelet for Nodes $U_{1}^{\prime}$

1: We partition the deployment square into squarelets, each with side length $r / \sqrt{5}$ (as in [18], see Figure 1 (b) for illustration). Thus, we have $\left\lceil\frac{a}{r / \sqrt{5}}\right\rceil$ squarelets. Each squarelet is denoted by $(i, j)$ when it is the $i$ th column and $j$ th row.

2: We build the Euclidean minimum spanning tree, denoted as $\operatorname{EMST}\left(U_{1}^{\prime}\right)$, connecting nodes in $U_{1}^{\prime}$, using following method (also described in Lemma 11):

(1) Originally, $k$ nodes $U_{1}^{\prime}$ form $k$ components;

(2) for the $g$ th step, where $g=1,2, \cdots, k-1$, partition the deployment square into at most $k-g$ square-shaped-cells, each with side length $\left\lceil\frac{a}{\lfloor\sqrt{k-g}\rfloor}\right\rceil$;

(3) find a cell that contains two nodes of $U_{1}^{\prime}$ that are from 2 different connected components and then connect them using Manhattan routing; merge these two connected components.

3: For each link $u v$ in the tree $\operatorname{EMST}\left(U_{1}^{\prime}\right)$, assume that $u$ and $v$ are inside squarelet $\left(i_{u}, j_{u}\right)$ and squarelet $\left(i_{v}, j_{v}\right)$ respectively. Find a node $w$ in squarelet $\left(i_{v}, j_{u}\right)$ (or squarelet $\left(i_{u}, j_{v}\right)$ ), i.e., $u w v$ is a Manhattan path connecting $u$ and $v$. We find the shortest path (with minimum Euclidean length) connecting $u w$ and $w v$ via the CDS constructed previously.

4: The resulted structure by uniting all such shortest paths for all links in $\operatorname{EMST}\left(U_{1}^{\prime}\right)$ will serve as multicast. Notice that here such structure may not be a tree. If this is the case, we could remove the cycles that do not contain nodes from $U_{1}^{\prime}$. Denote the resulted tree as $M T\left(U_{1}^{\prime}\right)$.

Using Manhattan path to connect nodes is to avoid the hot-spot area in the center of region produced by directed shortest path routing, via a kind of load balancing. Due to such pseudo-load-balancing approach, we can later show that the "load" (total data rates of all routing requests) on each node on CDS (equivalently each squarelet) is at most a fraction of $W$ almost surely.

To show that the above routing achieves the asymptotically optimum multicast capacity, we need show that the total number of data copies of a multicast bit is at most $O\left(\frac{r \cdot \sqrt{k} \cdot n}{a}\right)$, which will be derived based on the upper bound on the area covered by all transmission disks in the multicast tree $M T\left(U_{1}^{\prime}\right)$. We will prove that, with high probability, the tree $M T\left(U_{1}^{\prime}\right)$ has Euclidean length at most a constant factor of the Euclidean length of tree $\operatorname{EMST}\left(U_{1}^{\prime}\right)$.

THEOREM 19. With high probability, the total Euclidean length of the multicast tree $M T\left(U_{1}^{\prime}\right)$ is within a constant $c_{5}$ factor of $\left\|\operatorname{EMST}\left(U_{1}^{\prime}\right)\right\|$, i.e., $\left\|M T\left(U_{1}^{\prime}\right)\right\| \leq c_{5}\left\|\operatorname{EMST}\left(U_{1}^{\prime}\right)\right\|$.

PROOF. For $k$ nodes $U_{1}^{\prime}$ in a multicast session with source node $v_{1}$, we will first construct the Euclidean minimum spanning tree $\operatorname{EMST}\left(U_{1}^{\prime}\right)$. Then for each edge $u v$ in $\operatorname{EMST}\left(U_{1}^{\prime}\right)$, we will replace it with a Manhattan path $u w v$ Here node $w$ is in the same column with $v$ and same row with $u$ in the squarelet partition. The resulting structure is call Manhattan tree $M H\left(U_{1}^{\prime}\right)$. Then for each edge $u v$ in $M H\left(U_{1}^{\prime}\right)$, we find the shortest path, denoted as $\mathbf{P}_{G}(u, v)$, connecting them in the original communication graph $G$. Here we denote the final routing structure as $S T\left(U_{1}^{\prime}\right)$. We will prove that the total length of $S T\left(U_{1}^{\prime}\right)$ is a constant factor of the total length of $\operatorname{EMST}\left(U_{1}^{\prime}\right)$, with high probability.

For every edge $u v$ in the Manhattan routing structure, we describe a method to find a routing path connecting them. When 
$\|u-v\| \leq 1$, no additional node is needed. Otherwise, we will try to find a node $w$ satisfying the following conditions

1. $\|w-u\| \geq \sigma \cdot r$, for some small constant $\sigma>0$.

2. Node $w$ is close to the direction of $u v$, i.e., $\angle w u v \leq \varphi$ for some pre-defined angle $\varphi<\pi / 3$.

We can show that node $w$ makes a non-negligible progress, i.e., $\|v-u\|-\|v-w\| \geq \varrho \cdot r$ for some constant $\varrho>0$. After node $w$ is found, we then recursively find nodes to connect $w$ and $v$. Obviously, the area of the region $A$ to select $w$ is $\varphi \cdot\left(1-\sigma^{2}\right) r^{2}$ and the area of the deployment region is $a^{2}$. Let $\ell$ be the number of relay nodes we need to find to reach node $v$ from $u$ using the above approach. Then the overall probability that at least one such intermediate node $w$ used to connect $u$ and $v$ is empty is at most $\left(1-\frac{\varphi \cdot\left(1-\sigma^{2}\right)}{(a / r)^{2}}\right)^{n} \cdot \ell$. Then the probability that every time we can find a relay node is at least $1-\left(1-\frac{\varphi \cdot\left(1-\sigma^{2}\right)}{(a / r)^{2}}\right)^{n} \ell$. We denote the first relay node found by node $u$ as $w$. Then $\|u-v\|-\|v-w\|$ is at least $r-r \sqrt{1+\sigma^{2}-2 \sigma \cdot \cos \varphi}$. By induction, the number of relay nodes connecting $u$ and $v$ is at most $\ell \leq \frac{\|u-v\|}{r-r \sqrt{1+\sigma^{2}-2 \sigma \cdot \cos \varphi}}$. Thus, the probability that we can connect $u$ and $v$ using the above approach is at least $1-\left(1-\frac{\varphi \cdot\left(1-\sigma^{2}\right)}{(a / r)^{2}}\right)^{n} \cdot \frac{\|u-v\|}{r-r \sqrt{1+\sigma^{2}-2 \sigma \cdot \cos \varphi}}$. Let $\chi=\frac{\sqrt{2}}{1-\sqrt{1+\sigma^{2}-2 \sigma \cdot \cos \varphi}}$.

The probability we can find a sequence of nodes $w$ for all edges in $M H\left(U_{1}^{\prime}\right)$ based on previous approach is at least

$$
1-\left[1-\frac{\varphi \cdot\left(1-\sigma^{2}\right)}{(a / r)^{2}}\right]^{n} \cdot \frac{\sqrt{2}\left|\operatorname{EMST}\left(U_{1}^{\prime}\right)\right|}{r-r \sqrt{1^{2}+\sigma^{2}-2 \sigma \cdot \cos \varphi}} .
$$

Recall that, with high probability, we have $\left\|\operatorname{EMST}\left(U_{1}^{\prime}\right)\right\| \leq \tau$. $\sqrt{k} \cdot a$. Then, when $a \leq r \cdot \sqrt{\frac{c \cdot n}{\ln n}}$, the above probability is at least $1-\left(\frac{1}{n}\right)^{\frac{\varphi\left(1-\sigma^{2}\right)}{c}-\frac{1}{2}} \cdot \frac{1}{\sqrt{\ln n}} \cdot \sqrt{c} \cdot \chi \cdot \sqrt{k} \cdot \tau \geq 1-\left(\frac{1}{n}\right)^{\frac{\varphi\left(1-\sigma^{2}\right)}{c}-\frac{3}{2}}$. $\frac{1}{(\ln n)^{3 / 2}} \cdot c^{3 / 2} \cdot \chi \cdot \theta_{1} \cdot \tau$. The inequality comes from the fact that $k \leq \theta_{1} a^{2} / r^{2}$. To ensure that the above probability goes to 1 as $n \rightarrow \infty$, it is sufficient (and necessary) to require that

$$
\frac{\varphi\left(1-\sigma^{2}\right)}{c}-\frac{3}{2} \geq 0 .
$$

We can easily find such $\varphi, \sigma$ and $c$ to make it satisfiable. Notice that here we need $0<c<4 \pi / 9$. Thus, with high probability, the total length of $S T\left(U_{1}^{\prime}\right)$ has Euclidean length at most $\frac{\sqrt{2}\left\|E M S T\left(U_{1}^{\prime}\right)\right\|}{1-\sqrt{1+\sigma^{2}-2 \sigma \cdot \cos \varphi}}$. Because for any two nodes $u$ and $v$ in the net-

work, if $\|u-v\|>r$, then the shortest path connecting $u$ and $v$ via the CDS has length at most $c_{4} \leq 5$ times the length of the shortest path connecting them in the original random communication graph $G=(V, E)$, see [3] for proofs. Thus, with high probability, the total length of $S T\left(U_{1}^{\prime}\right)$ has Euclidean length at most $\frac{c_{4} \sqrt{2} \cdot\left\|E M S T\left(U_{1}^{\prime}\right)\right\|}{1-\sqrt{1+\sigma^{2}-2 \sigma \cdot c^{2}}}$

$1-\sqrt{1+\sigma^{2}-2 \sigma \cdot \cos \varphi}$

Recall that for CDS constructed previously, we know that the shortest Euclidean path connecting $u$ and $v$ via CDS is only at most 3 times of the length of the shortest path connecting $u$ and $v$ in the network $G=(V, E)$. Thus, the multicast tree $M T\left(U_{1}^{\prime}\right)$ based on CDS has Euclidean length at most $3\left\|M T\left(U_{1}^{\prime}\right)\right\|$. The theorem follows by letting $c_{5}=3 \frac{c_{4} \sqrt{2}}{1-\sqrt{1+\sigma^{2}-2 \sigma \cdot \cos \varphi}}$.

Consequently, we have Euclidean length $\left\|M T\left(U_{1}^{\prime}\right)\right\|<c_{5} \eta \sqrt{k}$. $a$ with high probability since $\left\|\operatorname{EMST}\left(U_{1}^{\prime}\right)\right\| \leq \eta \sqrt{k} \cdot a$ for $\eta=$ $2 \sqrt{2}$ (see Lemma 11). Here we denote the region covered by all transmission disks of all internal nodes in $M T\left(U_{1}^{\prime}\right)$ as $D(T)$ and the number of nodes lying in $D(T)$ as $C$. We then show that with high probability, the multicast capacity achieved using above routing approach is within a constant factor of the asymptotic optimum. We essentially show that, with high probability, the number $C$ of nodes that will receive a copy of the multicast data is within $2 E(C)$.

THEOREM 20. The total multicast capacity $\Lambda_{k}(n)$ achievable by all multicast flows is at least $c_{6} \frac{a \cdot W}{r \sqrt{k}}$, when $k \leq \theta_{1} \frac{a^{2}}{r^{2}}$ and $a / r \leq$ $\sqrt{\frac{c n}{\ln n}}$ for some constant $c \in(0,4 \pi / 9)$. Here $c_{6}$ is a constant.

Proof. Consider a set of receivers $U_{1}$ for source node $v_{1}$. Let tree $T$ be the multicast tree $M T\left(U_{1}^{\prime}\right)$ constructed above. Let $X_{i} \in$ $\{0,1\}$ be an indicator variable whether the $i$ th node $v_{i}$ will fall inside the region $D(T)$ for a multicast tree $T$. Clearly $p=\operatorname{Pr}\left(X_{i}=1\right)=$ $\frac{|D(T)|}{a^{2}}$. Notice that the area of $D(T)$ is at most $2 r \cdot\|T\|+k \pi r^{2} / 2$, and edge length $\|T\| \leq c_{5} \eta \cdot \sqrt{k} \cdot a$ with high probability. Obviously, $X=\sum_{i=1}^{n} X_{i}$ is the number of nodes falling inside the region $D(T)$, and $X$ is binomial distribution. Using Lemma 3,

$$
\begin{aligned}
& \operatorname{Pr}\left(C>|D(T)| \cdot \frac{2 n}{a^{2}}\right) \leq \frac{|D(T)| \cdot \frac{2 n}{a^{2}} \cdot\left(1-\frac{|D(T)|}{a^{2}}\right)}{\left(|D(T)| \cdot \frac{2 n}{a^{2}}-\frac{n \cdot|D(T)|}{a^{2}}\right)^{2}} \\
= & \frac{2\left[1-\frac{|D(T)|}{a^{2}}\right]}{|D(T)| \cdot \frac{n}{a^{2}}} \leq \frac{2 a^{2}}{n \cdot|D(T)|} \leq \frac{2 c_{0} \cdot a^{2}}{n \cdot \tau \sqrt{k} \cdot a \cdot r} \\
= & \frac{a}{r} \frac{1}{n \sqrt{k}} \frac{2 c_{0}}{\tau} \leq \frac{1}{\sqrt{n \cdot k \cdot \ln n}} \frac{2 c_{0} \sqrt{c}}{\tau}
\end{aligned}
$$

The last inequality comes from the assumption that $a / r \leq \sqrt{\frac{c \cdot n}{\ln n}}$. The second to last inequality comes from Lemma 12 that $|D(T)| \geq$ $\frac{\tau \sqrt{k} \cdot a \cdot r}{c_{0}}$. Consequently, $\operatorname{Pr}\left(C \leq|D(T)| \cdot \frac{2 n}{a^{2}}\right) \geq 1-\frac{1}{\sqrt{n \cdot k \cdot \ln n}} \frac{2 c_{0} \sqrt{c}}{\tau}$. Thus the number of nodes that can get a copy of the data for multicast within nodes $U_{1}^{\prime}$, with high probability, is at most

$$
|D(T)| \cdot \frac{2 n}{a^{2}} \leq c_{5} \eta \sqrt{k} \cdot \frac{4 n \cdot r}{a}+\pi n k \frac{r^{2}}{a^{2}} \leq\left(4 c_{5} \eta+\pi \sqrt{\theta_{1}}\right) \cdot n \sqrt{k} \cdot \frac{r}{a},
$$

The last inequality comes from $k \leq \theta_{1} \frac{a^{2}}{r^{2}}$.

Recall that, by performing multicast based on CDS structure, we can guarantee that each node will be able to transmit once every $\Delta+1$ time-slots. This implies that the total bits/sec achieved by all nodes is at least $n \cdot W /(\Delta+1)$. Consequently, the multicast capacity is at least $\frac{n \cdot W /(\Delta+1)}{\left(4 c_{5} \eta+\pi \sqrt{\theta_{1}}\right) \cdot n \sqrt{k} \cdot \frac{r}{a}}=\frac{1}{\left(4 c_{5} \eta+\pi \sqrt{\theta_{1}}\right) \cdot(\Delta+1)} \cdot \frac{a \cdot W}{r \sqrt{k}}$. This finishes the proof by setting $c_{6}=\frac{1}{\left(4 c_{5} \eta+\pi \sqrt{\theta_{1}}\right) \cdot(\Delta+1)}$.

Observe that the correctness of Theorem 20 relies on the fact that $\frac{a}{r} \leq \sqrt{\frac{c n}{\ln n}}$ and $k \leq \theta_{1} a^{2} / r^{2}$. Here constant $0<c<4 \pi / 9$. Consequently, by letting $\frac{a}{r}=\sqrt{\frac{c n}{\ln n}}$ for $0<c<4 \pi / 9$, and $c_{2}^{\prime}=c_{6} \sqrt{c}$, based on Theorem 20, we have

COROLLARY 21. The multicast capacity for a random network of $n$ nodes, when $k<\theta_{1} \cdot a^{2} / r^{2}$, is at least

$$
\Lambda_{k}(n) \geq c_{2}^{\prime} \cdot \frac{\sqrt{n}}{\sqrt{\log n} \cdot \sqrt{k}} \cdot W=\Omega\left(\frac{\sqrt{n}}{\sqrt{\log n} \cdot \sqrt{k}} \cdot W\right) .
$$

The multicast capacity per node (with $n$ sources) is

$$
\lambda_{k}(n)=\frac{\Lambda_{k}(n)}{n}=\Omega\left(\frac{1}{\sqrt{n \log n} \cdot \sqrt{k}} \cdot W\right) .
$$

Observe that the correctness of Theorem 20 requires that the "load" of every routing node is no more than a constant factor of $W$ bits/sec, due to the requirement of TDMA node scheduling. Unfortunately, it is unknown now whether we can prove whether such 
condition is satisfied with high probability. We will prove a weaker capacity lower bound: with high probability, the traffic load on any routing node and all interfering nodes is no more than $O(W)$.

Given a squarelet, we define its load as the total number of multicast sessions that will be routed through nodes inside this squarelet. We show that under our routing algorithm, for any squarelet, with high probability, its load is no more than $\Theta(\sqrt{k n \log n})$. To prove our claim, we first study a simple unicast case. Consider a grid of $L \times L$ squarelets. Consider a specific squarelet $\mathbf{s}$ that is of $i$ th row and $j$ th column in the squarelet-grid. Randomly pick two nodes $u$ and $v$ from the grid and connect them via Manhattan routing. Let $p_{\mathbf{s}}(L)$ denote the probability that the Manhattan routing will use nodes from the squarelet $\mathbf{s}$. Then

$$
p_{\mathbf{s}}(L)=\frac{i-1}{L^{2}} \cdot \frac{L-i+1}{L}+\frac{j-1}{L^{2}} \cdot \frac{L-j+1}{L} .
$$

Here $\frac{i-1}{L^{2}} \cdot \frac{L-i+1}{L}$ (resp. $\frac{j-1}{L^{2}} \cdot \frac{L-j+1}{L}$ ) is the probability that squarelet $\mathbf{s}$ is used when $u$ (resp. $v$ ) is on the same row (resp. column) as s. It is easy to show that $\frac{2}{L^{2}} \leq p_{\mathbf{s}}(L) \leq \frac{2}{L}$.

Let us now study the number of times that a specific squarelet $\mathbf{s}$ is used by our routing structure for multicast. Recall that we will construct the Euclidean minimum spanning tree as the method described in Lemma 11 and then find multicast routing structure as Algorithm 1. For a given multicast session, this squarelet $\mathbf{s}$ may be used in any one of the $k$ steps to build the spanning tree. For step $g$ (with $1 \leq g \leq k-1$ ), recall that we will partition the square with side-length $a$ into $\lfloor\sqrt{k-g}\rfloor^{2} \leq k-g$ cells, each with side-length $\frac{a}{\lfloor\sqrt{k-g}\rfloor}$. From pigeonhole principle, there exists a cell that contains two nodes, say $u$ and $v$, from two different connected components. We will connect them and merge these two connected components. Here we will connect $u$ and $v$ using Manhattan routing as illustrated in Figure 2. Let $X_{\mathbf{s}, g}$ be the indicator whether the specific squarelet $\mathbf{s}$ is used in this $g$ th step. Clearly,

$$
\operatorname{Pr}\left(X_{\mathbf{s}, g}=1\right)=\frac{1}{\lfloor\sqrt{k-g}\rfloor^{2}} \cdot p_{\mathbf{s}}\left(\left\lceil\frac{\frac{a}{\lfloor\sqrt{k-g}\rfloor}}{r / \sqrt{5}}\right\rceil\right),
$$

where $\frac{1}{\lfloor\sqrt{k-g}\rfloor^{2}}$ is the probability that the cell containing squarelet $\mathbf{s}$ is used, and $p_{\mathbf{s}}\left(\left\lceil\frac{\frac{a}{\lfloor\sqrt{k-g}\rfloor}}{r / \sqrt{5}}\right\rceil\right)$ is the probability that $\mathbf{s}$ is used when that cell containing $\mathbf{s}$ is used. Here $\left\lceil\frac{\frac{a}{\lfloor\sqrt{k-g}\rfloor}}{r / \sqrt{5}}\right\rceil$ is the number of squarelets per row in a cell, i.e., the value of $L$ in formula 8 . Thus,

$$
\begin{aligned}
p & =\operatorname{Pr}\left(X_{\mathbf{s}}=1\right) \leq \sum_{g=1}^{k-1} \operatorname{Pr}\left(X_{\mathbf{s}, g}=1\right) \\
& =\sum_{g=1}^{k-1} \frac{1}{\lfloor\sqrt{k-g}\rfloor^{2}} \cdot p_{\mathbf{s}}\left(\left\lceil\frac{\frac{a}{\lfloor\sqrt{k-g}\rfloor}}{r / \sqrt{5}}\right\rceil\right) \leq \frac{4 \sqrt{10}}{5} \sqrt{k} \cdot \frac{r}{a}
\end{aligned}
$$

Notice that, to achieve larger multicast capacity, we will set $\frac{a}{r}=$ $\sqrt{\frac{c n}{\ln n}}$ for some constant $0<c<4 \pi / 9$ (see proof of Theorem 19). Thus, $\operatorname{Pr}\left(X_{\mathrm{s}}=1\right) \leq \frac{4 \sqrt{10}}{5} \sqrt{\frac{k \cdot \ln n}{c n}}$. Then we have

LEMMA 22. Given $n_{s}$ multicast sessions, the expected number of multicast routing flows that use a specific squarelet $\mathbf{s}$ is at most $\frac{4 \sqrt{10}}{5 \sqrt{c}} \cdot n_{s} \cdot \sqrt{\frac{k \cdot \ln n}{n}}$. When $n_{s}=n$, it is at most $\frac{4 \sqrt{10}}{5 \sqrt{c}} \cdot \sqrt{k \cdot n \cdot \ln n}$.

Recall that all $n$ multicast sessions will randomly select its receivers. Let $\mu=\frac{4 \sqrt{10}}{5 \sqrt{c}} \cdot \sqrt{k \cdot n \cdot \ln n}$. Using Lemma 3, we can show that with probability at least $1-\frac{2(1-p)}{\mu^{2}}=1-O\left(\frac{1}{\sqrt{k n \log n}}\right)$ the number of multicast flows routing through a squarelet is at most $2 \mu$. Thus, we have the following theorem
THEOREM 23. With probability at least $1-O\left(\frac{1}{\sqrt{k n \log n}}\right)$, the number of multicast flows that pass through a squarelet is at most $2 \mu=\frac{8 \sqrt{10}}{5 \sqrt{c}} \cdot \sqrt{k \cdot n \cdot \ln n}=c_{11} \sqrt{k n \log n}$.

Thus, by letting $\lambda_{i}=\frac{c_{12} W}{\sqrt{k n \log n}}, 1 \leq i \leq n$, for some proper constant $0<c_{12}<1$, then with high probability, the total traffic load at any specific squarelet is at most $c_{11} c_{12} W$ bits/sec. Since the size of the squarelet is $r / \sqrt{5}$ and the interference range $R=\Theta(r)$, we can show that the total flow requirements of all squarelets that could cause interference to a given squarelet is also at most $\Delta$. $c_{11} c_{12} W$ with high probability. Here $\Delta=\Theta(1)$ is the number of squarelets within distance $R$ from this squarelet. Thus, by choosing $c_{12} \leq \frac{1}{\Delta c_{11}}$, we can schedule flows at this squarelet with high probability (at least $1-O\left(\frac{1}{\sqrt{k n \log n}}\right)$ ). Recall that w.h.p., a flow will pass through $O(\sqrt{k} \cdot a / r)=O(\sqrt{k n / \log n})$ squarelets. Then the probability that a given flow can be scheduled via TDMA is at least $\left(1-O\left(\frac{1}{\sqrt{k n \log n}}\right)\right)^{O(\sqrt{k n / \log n})}=1-\frac{O(1)}{\log n}$.

\subsection{When $k=\Omega\left(\frac{a^{2}}{r^{2}}\right)$}

In this case, we have proved that the upper bound on the total multicast capacity is only $\Theta(W)$. Obviously, the total multicast capacity is at least the lower bound of the capacity for broadcast. In [11], they present a broadcast scheme to achieve capacity $\Theta(W)$. Thus, we have the following theorem

THEOREM 24. The total multicast capacity $\Lambda_{k}(n)$ achievable by all multicast flows is at least $c_{7} W$ when $k=\Omega\left(a^{2} / r^{2}\right)$, where $c_{7}=\frac{1}{\Delta+1}$ and constant $\Delta$ is the maximum number of CDS nodes that are within interference range $R$ of a node.

\section{OTHER MULTICASTS}

\subsection{Capacity Bound for Group Multicast}

In previous sections we have studied the asymptotic multicast capacity by assuming that we randomly select $k-1$ receivers for each multicast session. In this section, we study the multicast capacity of so-called $k$-group multicast: for each source node $v_{i}$, there are $k-1$ groups of receivers $g_{i, 1}, g_{i, 2}, \cdots, g_{i, k-1}$. The receivers in each group $g_{i, j}$ are covered by a disk with radius $\delta \cdot r$ for a constant $\delta$ and centered at one of the receivers in the group. We assume that the center node in each group is randomly selected. The number of nodes in each group could be arbitrary. For simplicity, let node $z_{i, j}$ be the center node of group $g_{i, j}$. We then study the multicast capacity for group-multicast when each node $v_{i}$ will have $k-1$ randomly selected groups and it wants to send data with rate $\lambda_{i}$ to all receivers in these $k-1$ groups.

As the case when each group has only one node, when $k \geq$ $\theta_{1} a^{2} / r^{2}$, it is easy to prove that the capacity for group-multicast is at most $W / \varrho_{2}$ as Theorem 18. Clearly, a simple broadcast based on the connecting dominating set constructed previously will also achieve a capacity for group-multicast at least $\frac{W}{\Delta+1}$. Consequently, we have

THEOREM 25. For group-multicast, when $k \geq \theta_{1} a^{2} / r^{2}$ for any constant $\theta_{1}>0$, the capacity of group-multicast is at most $W / \varrho_{2}$ and at least $\frac{W}{\Delta+1}$.

First, for group-multicast, a multicast tree has to reach the center node $z_{i, j}$ of each group $g_{i, j}$. Then from Theorem 14 , the capacity of group-multicast is at most $c_{1} \cdot \frac{a W}{r \sqrt{k}}$ with high probability when $k<\theta_{1} \cdot a^{2} / r^{2}$. We then show how to design multicast routing for the group-multicast problem: we first apply our 
multicast scheme for traditional multicast when nodes $z_{i, j}, 1 \leq$ $j \leq k-1$, are receivers for source node $v_{i}$. We then let node $z_{i, j}$ multicast locally to all receivers in the group $g_{i, j}$. We already proved in Theorem 19, the total length of the multicast tree to span these randomly selected nodes $z_{i, j}$ is at most $c_{5}\left\|E M S T\left(U_{i}^{\prime}\right)\right\|$ with high probability. Recall that $\left\|\operatorname{EMST}\left(U_{i}^{\prime}\right)\right\| \leq \frac{3 \tau(2) \sqrt{k} \cdot a}{2}$ with high probability. Notice that the total area covered by transmitting disks of relay nodes used for relaying data from each $z_{i, j}$ to receivers in its group is at most $\pi(\delta+1) r^{2}$. Then, the area covered by all transmitting disks for a multicast session is at most $2 r \cdot\left|M T\left(U_{1}^{\prime}\right)\right|+(k-1) \cdot \pi(\delta+1) r^{2}$, which is w.h.p.at most $2 r \cdot c_{5} \frac{3 \tau(2) \sqrt{k} \cdot a}{2}+k \cdot \pi(\delta+1) r^{2} \leq\left(\theta_{1} \pi(\delta+1)^{2}+3 c_{5} \tau(2)\right)$. $\sqrt{k} \cdot a \cdot r$. The last inequality comes from the fact that $k \leq \theta_{1} \frac{a^{2}}{r^{2}}$. For convenience, let $c_{8}=\theta_{1} \pi(\delta+1)^{2}+3 c_{5} \tau(2)$. Then similar to Theorem 20, we can prove that the number of nodes that will get a copy of the data from one multicast session is at most $c_{8} \sqrt{k} \cdot a \cdot r \cdot \frac{2 n}{a^{2}}=2 c_{8} \cdot n \cdot \sqrt{k} \cdot \frac{r}{a}$. Thus, we have

THEOREM 26. When $k \leq \theta_{1} a^{2} / r^{2}$, the aggregated multicast capacity for group-multicast with $k-1$ groups is at most $c_{1} \cdot \frac{a W}{r \sqrt{k}}$, and is at least $c_{9} \frac{a \cdot W}{r \sqrt{k}}$, with high probability. Here constant $c_{9}=$ $2 c_{8}(\Delta+1)$.

\subsection{Bounds for Arbitrary Networks}

In previous studies we concentrated on the multicast capacity for random networks when nodes will be randomly placed in the deployment region. In this section we will study what is the asymptotic maximum multicast capacity that can be achieved by a specific connected network when nodes' position can be carefully selected.

We first present a constructive lower bound on the multicast capacity. Assume that $n$ nodes are deployed in a $\sqrt{n}$ by $\sqrt{n}$ grid, each cell has side-length $r$, i.e. the side-length of the square is $a=r \sqrt{n}$. The $k-1$ receivers are randomly selected from the grid points. We then perform multicast as before: the multicast tree is constructed based on the Euclidean minimum spanning tree connecting source node and $k-1$ receivers. Let $L$ be the total length of the Euclidean MST constructed above. Similar to previous studies in Subsection 4.2, we know that the multicast capacity $\Lambda_{n}$ satisfies $\Lambda_{n} \geq c_{6}^{\prime} \frac{W \cdot a^{2}}{L \cdot r}$ for some constant $c_{6}^{\prime}$ depending only on $R / r$. Lemma 11 gives an upper bound on $L$ for the Euclidean minimum spanning tree. This implies the following corollary:

COROLlARY 27. The multicast capacity $\Lambda_{n}$ for an arbitrary network (we can choose node positions), is at least $\frac{c_{6}^{\prime}}{2 \sqrt{2}} \frac{\sqrt{n}}{\sqrt{k}} \cdot W$.

\section{LITERATURE REVIEWS}

Network capacity has been extensively studied recently. For a given statistical description of the network, a set of constraints (such as power per node, link capacity, etc.), and a list of desired communication pairs, the capacity region is the closure of all rate tuples that can be achieved simultaneously. Here a rate tuple specifies the rate for each of the desired communications. Kyasanur and Vaidya [13] studied the capacity region on given multi-hop multi-radio multi-channel wireless networks when there are total $c$ channels available and each node has $m$ wireless interfaces with $m \leq c$. On the other aspect, several papers $[2,12]$ recently studied how to satisfy a certain traffic demand vector from all wireless nodes by a joint routing, link scheduling, and channel assignment under certain wireless interference models.

Gupta and Kumar [7] studied the asymptotic unicast capacity of random multi-hop wireless networks for two different models. When each wireless node is capable of transmitting at $W$ bits per second using a constant transmission range, the throughput obtainable by each node for a randomly chosen destination is $\Theta\left(\frac{W}{\sqrt{n \log n}}\right)$ bits per second under the protocol-interference model, where $n$ in number of nodes. If nodes are optimally assigned and transmission range is optimally chosen, even under optimal circumstances, the throughput is only $\Theta\left(\frac{W}{\sqrt{n}}\right)$ bits per second for each node. Similar results also hold for physical interference model. Grossglauser and Tse [6] recently showed that mobility actually can help to improve the unicast capacity if we allow arbitrary large delay. Their main result shows that the average long-term throughput per source-destination pair can be kept constant even as the number of nodes per unit area increases with the aid of mobility and dynamic power adjustment. Notice that this is in sharp contrast to the fixed network scenario (when nodes are static after random deployment). The main idea used in [6] is to use some intermediate node to serve as ferry node: this node will carry the data from the source node and move around and it will dump the data to the target node when it is within its communication range. In other words, essentially, the result presented in [6] still obey the capacity bound proposed in [7]: the capacity is improved because the average distance $\bar{L}$ a packet has to be transmitted is reduced from $\Theta(1)$ in [7] to $\Theta(r(n))$ in [6]. In summary, for random networks, under the protocol model, the achievable per-node throughput capacity $\lambda(n)$ and the average travel distance $\bar{L}$ satisfies $\lambda(n) \cdot \bar{L} \leq \Theta\left(\frac{W}{\Delta^{2} n \cdot r(n)}\right)$. Similar phenomenon has also been observed in [14].

Broadcast capacity of an arbitrary network has been studied in $[11,20]$. They essentially showed that the broadcast capacity of a given network is $\Theta(W)$ for single source broadcast and the achievable broadcast capacity per node is only $\Theta(W / n)$ if each of the $n$ nodes will serve as source node. The upper bound $\Theta(W)$ on broadcast capacity trivially holds since each node can receive at most $W$ bits/sec. The capacity $\Theta(W)$ is achieved by constructing a connected dominating set in which we can schedule every node in CDS to transmit at least once in constant time slots. This capacity bounds also apply to random networks. Keshavarz-Haddad et $a l$. [10] studied the broadcast capacity with dynamic power adjustment for physical interference model.

Multicast capacity was not fully studied in the literature. Jacquet and Rodolakis [9] studied the scaling properties of multicast for random wireless networks. They essentially studied the normalized multicast cost, which is defined as the ratio of the number of links in the multicast tree over the average route length from a random source in the multicast group to a random destination in the multicast group. They briefly showed that the maximum rate at which a node can transmit multicast data is $O\left(\frac{W}{\sqrt{k n \log n}}\right)$. At the same time as our results, Shakkottai et al. [18] studied the multicast capacity of random networks when the number of multicast sources is $n^{\epsilon}$ for some $\epsilon>0$, and the number of receivers per multicast flow is $n^{1-\epsilon}$. They assume the protocol interference model and use the dense random network model. They show that the sum of the source rates $\Lambda(n)$ that the network can support is $O\left(\frac{\sqrt{n^{\epsilon}}}{\sqrt{\log n}}\right)$ w.h.p., with a per flow throughput capacity of $O\left(\frac{1}{\sqrt{n^{\epsilon} \log n}}\right)$ w.h.p.. Notice that this result can be implied by our results using $n_{s}=n^{\epsilon}$ and $k=n^{1-\epsilon}$. To achieve the upper bound, they propose a simple and novel routing architecture, called the multicast comb, to transfer multicast data in the network.

In [7], the capacity of wireless networks are solved under a number of assumptions, among them point-to-point coding which excludes for example the multi-access and broadcast codes. Gastpar and Vetterli [5] and Liu et al. [15] studied the capacity of wireless networks when network coding can be used to improve the capacity. Gastpar and Vetterli demonstrated the power of network cod- 
ing: under the point-to-point coding assumption considered in [7], the achievable data rate is constant, independent of the number of nodes. Liu et al. [15] showed that for multi-pair unicast traffic in wireless multi-hop networks, the benefit of network coding and broadcasting on the concurrent throughput rate is upper bounded by a constant factor for both the protocol model and the physical model. This is true for randomly deployed networks.

\section{CONCLUSIONS}

In this paper, we derived matching analytical upper bounds and lower bounds on multicast capacity of a wireless network when all nodes are uniformly and randomly deployed in a square region with side-length $a$, and all nodes have the same transmission range $r$. Observe that all our results are proved when the deployment region is a square with side-length $a$ and the transmission ranges of all nodes are uniform with value $r$. We can show that all our results still apply when the deployment region is a square with side length $a=1$, while the transmission range is selected appropriately, i.e., $r=\Theta\left(\sqrt{\frac{\log n}{\pi n}}\right)$. We can show that our results still hold when $r=1$ while the deployment region has a bounded aspect ratio such as a disk. Further, we considered the protocol interference model for random networks. We can show that our results still hold (with different constants) when we apply the physical interference model (where all nodes have fixed uniform transmission power $P$ and fixed minimum SINR threshold) and the signal power at distance $d$ decays as $\frac{1}{d^{\alpha}}$ for $\alpha>2$. The basic idea is to show that, for such physical interference model, there is a logic transmission range $r$ and interference range $R$ (with $R=\Theta(r)$ ) such that when $\|u-v\| \leq r$ and no other transmitting nodes within distance $R$ of receiving node $v$, node $u$ can always successfully send data to $v$. All computations will be similar by using such logic transmission range and interference range. The details of all computations are omitted here due to space limit.

Notice that the results presented here did not consider the additional burden in coordinating access to wireless channels, the effect of mobility and link failures, the effect of the need to route traffic in a distributed way. We also did not address the delay of the route. The delay could be caused by burst traffic or when nodes are mobile and links are not stable. It can also be imagined that using directional antennas or beam-forming will help to improve the spatially concurrency of transmissions and thus the capacity of the networks.

There are some interesting questions left for study for multicast capacity. The first question is what is the multicast capacity when the link capacity is not uniform: shorter links will have larger capacity. The second question is what is the multicast capacity when the Gaussian channel is used, instead of assuming that each node has a constant transmission range and has a constant data rate $W$. Last but not the least question is what is the tradeoffs between the delay and multicast capacity for random mobile networks?

\section{ACKNOWLEDGMENT}

The authors are grateful for a variety of valuable comments from the anonymous reviewers and the shepherd Dr. Ben Liang, which enable us to improve the paper in a number of different ways. We are also grateful to Y.-W. Wu, K. Moaveninejad, X.-F. Mao, P. Xu for fruitful discussions during the preliminary state of the paper.

\section{REFERENCES}

[1] Ahn, G.-S., Miluzzo, E., Campbell, A. T., Hong, S. G., AND CuOMO, F. Funneling-mac: A localized, sink-oriented mac for boosting fidelity in sensor networks. In ACM SenSys (2006).
[2] Alicherry, M., Bhatia, R., AND Li, L. E. Joint channel assignment and routing for throughput optimization in multi-radio wireless mesh networks. In ACM MobiCom (2005), pp. 58-72.

[3] Alzoubi, K., LI, X.-Y., WANG, Y., WAN, P.-J., AND Frieder, O., Geometric spanners for wireless ad hoc networks. IEEE Trans. on Parallel and Distri. Processing 2003 (Vol. 14, No. 4), pages 408-421.

[4] Du, D.-Z., AND Hwang, F.-K. A Proof of the Gilbert-Pollak Conjecture on the Steiner Ratio Algorithmica, 7(2,3), pp. 121-135 (1992).

[5] GastPar, M., AND VetTerli, M. On the capacity of wireless networks: the relay case. In IEEE INFOCOM (2002).

[6] Grossglauser, M., AND TSE, D. Mobility increases the capacity of ad-hoc wireless networks. In INFOCOMM (2001), vol. 3, pp. $1360-1369$.

[7] Gupta, P., AND Kumar, P. Capacity of wireless networks. IEEE Trans. on Information Theory, Volume 46, Issue 2, Mar 2000, Page(s):388-404.

[8] Gupta, P., And Kumar, P. R. Critical power for asymptotic connectivity in wireless networks. Stochastic Analysis, Control, Optimization and Applications: A Volume in Honor of W.H. Fleming, W. M. McEneaney, G. Yin, and Q. Zhang (Eds.) (1998).

[9] JACQUeT, P. AND Rodolakis G. Multicast Scaling Properties in Massively Dense Ad Hoc Networks, ICPADS '05: 11th Int. Conf. on Parrallel and Distri. Syst. - Workshops, 2005, pages 93-99.

[10] Keshavarz-Haddad, A. AND Riedi R. On the Broadcast Capacity of Multihop Wireless Networks: Interplay of Power, Density and Interference, 4th IEEE SECON, June 2007.

[11] Keshavarz-Haddad, A., Ribeiro, V., And Riedi, R. Broadcast capacity in multihop wireless networks. In ACM MobiCom 2006, pp. 239-250.

[12] Kodialam, M., AND NANDAGOPAL, T. Characterizing achievable rates in multi-hop wireless networks: the joint routing and scheduling problem. In ACM MobiCom 2003, pp. 42-54.

[13] Kyasanur, P., And Vaidya, N. H. Capacity of multi-channel wireless networks: impact of number of channels and interfaces. In ACM MobiCom 2005, pp. 43-57.

[14] Li, J., Blake, C., Couto, D. S. J. D., Lee, H. I., AND Morris, R. Capacity of ad hoc wireless networks. In ACM MobiCom (2001).

[15] LiU, J. Goeckel, D. Towsley, D. Bounds on the Gain of Network Coding and Broadcasting in Wireless Networks IEEE INFOCOM 2007. May 2007, pages 724-732.

[16] Penrose, M. The longest edge of the random minimal spanning tree. Annals of Applied Probability 7 (1997), 340-361.

[17] SAnti, P., AND Blough, D. M. The critical transmitting range for connectivity in sparse wireless ad hoc networks. IEEE Trans. on Mobile Computing 2 (Mar. 2003), 25-39.

[18] Shakkottai S., LiU X. And SRIKant R., The multicast capacity of ad hoc networks, Proc. ACM Mobihoc, 2007.

[19] STEELE, J. M. Growth rates of Euclidean minimal spanning trees with power weighted edges. The Annals of Probability 16, 4 (Oct 1988), 1767-1787.

[20] TAVLI, B. Broadcast capacity of wireless networks. IEEE Communication Letters 10, 2 (February 2006).

[21] Wan, P.-J., Alzoubi, K. M., AND Frieder, O. Distributed construction of connected dominating set in wireless ad hoc networks. In IEEE INFOCOM (2002).

[22] WANG, Y., LI, X.-Y., AND FRIEDER O. Distributed Spanner with Bounded Degree for Wireless Ad Hoc Networks Int. Journal on Found. of Compt. Sci., Vol. 14, No. 2 (2003) Pages 183-200.

[23] W. Wang, Y. Wang, X.-Y. Li, et al. Efficient Interference-Aware TDMA Link Scheduling for Static Wireless Networks, In ACM MobiCom, 2006.

[24] XUe, F., AND Kumar, P. R. The number of neighbors needed for connectivity of wireless networks. Wireless Networks, Volume 10, Issue 2, (March 2004), Pages 169-181.

[25] ZHENG, R. Information dissemination in power-constrained wireless networks. In IEEE INFOCOM (2006). 